\title{
ON PAIRS OF RECURSIVELY ENUMERABLE DEGREES
}

\author{
BY
}

\author{
KLAUS AMBOS-SPIES
}

\begin{abstract}
Lachlan and Yates proved that some, but not all, pairs of incomparable recursively enumerable (r.e.) degrees have an infimum. We answer some questions which arose from this situation. We show that not every nonzero incomplete r.e. degree is half of a pair of incomparable r.e. degrees which have an infimum, whereas every such degree is half of a pair without infimum. Further, we prove that every nonzero r.e. degree can be split into a pair of r.e. degrees which have no infimum, and every interval of r.e. degrees contains such a pair of degrees.
\end{abstract}

Lachlan [5] and, independently, Yates [11] have proved that the upper semilattice $(\mathbf{R}, \leqslant, \cup)$ of r.e. degrees is not a lattice: There are pairs of incomparable r.e. degrees which do not have an infimum. On the other hand, they have shown that for some incomparable r.e. degrees $\mathbf{a}_{0}$ and $\mathbf{a}_{1}, \mathbf{a}_{0} \cap \mathbf{a}_{1}$ exists.

Here we answer some questions which arose from the situation that some, but not all, pairs of r.e. degrees have an infimum. By constructing an incomplete strongly noncappable degree we first positively answer a question of Soare [10], whether there is an r.e. degree $\neq \mathbf{0}, \boldsymbol{0}^{\prime}$ which is not half of a pair of incomparable r.e. degrees which have an infimum. We then show that for every r.e. degree $\mathbf{a} \neq \mathbf{0}, \mathbf{0}^{\prime}$ there is a strongly noncappable degree incomparable with $\mathbf{a}$, and thereby deduce that every nonzero incomplete r.e. degree is half of a pair of r.e. degrees without infimum. This answers a question of Jockusch [4]. ${ }^{1}$ In [2] we had obtained partial answers to these questions by extending Lachlan's nondiamond technique of [5].

From the construction of a strongly noncappable degree we extract a new easy construction of a pair of r.e. degrees without infimum. We combine this construction with Sacks' splitting and density theorems to show that every r.e. degree can be split into a pair of r.e. degrees without infimum, and every proper interval of r.e. degrees contains such a pair of degrees.

It is pointed out how the above results imply and extend a result of Cooper [3] on minimal upper bounds for ascending sequences of uniformly r.e. degrees.

0. Preliminaries. Our notation, with a few exceptions, is that of Soare [10]. Lower case letters denote elements of $\omega$, the set of nonnegative integers; capital letters denote subsets of $\omega$. The letters $f, g, h$ stand for functions from $\omega$ to $\omega$. A set and its characteristic function are identified; i.e., $x \in A$ iff $A(x)=1$ and $x \notin A$ iff $A(x)=0$. $A \uparrow x$ is the restriction of $A$ to numbers less than $x . \mathbf{a}, \mathbf{b}, \mathbf{c}, \ldots$ denote recursively

Received by the editors May 28, 1982 and, in revised form, February 17, 1983.

1980 Mathematics Subject Classification. Primary 03D25.

${ }^{1}$ L. Harrington has independently answered these questions of Jockusch and Soare.

(C) 1984 American Mathematical Society $0002-9947 / 84 \$ 1.00+\$ .25$ per page 
enumerable degrees. $u(e, A, x)$ is the use function of $\{e\}^{A}(x)$; i.e., if $\{e\}^{A}(x) \downarrow$, then the computation of $\{e\}^{A}(x)$ uses only numbers less than $u(e, A, x)>0$, and if $\{e\}^{A}(x) \uparrow$, then $u(e, A, x)=0$. The use function of $\{e\}_{s}^{A}(x)$ is denoted by $u(e, A, x, s)$. We assume that for all $e, s, x$ and $A$,

$$
\{e\}_{s}^{A}(x) \downarrow \rightarrow e, x, u(e, A, x, s)<s .
$$

We fix a recursive 1-1 function $\langle x, y\rangle$ from $\omega \times \omega$ onto $\omega$ which is monotonic in both arguments. $(\langle x, y\rangle)_{0}=x$ and $(\langle x, y\rangle)_{1}=y$. For $n \geqslant 2,\left\langle x_{0}, \ldots, x_{n}\right\rangle=$ $\left\langle x_{0},\left\langle x_{1}, \ldots, x_{n}\right\rangle\right\rangle$.

$$
\begin{gathered}
A^{(x)}=\left\{y \in A:(y)_{0}=x\right\}, \\
A^{(x, y)}=\{w \in A: \exists z(w=\langle x, y, z\rangle)\}
\end{gathered}
$$

(note that $A^{(x, y)} \subseteq A^{(x)}$ ),

$$
A^{(<x)}=\bigcup_{y<x} A^{(y)}, \text { etc. }
$$

If in the following an r.e. set $X\left(X_{j}\right)$ is constructed in infinitely many stages, $X_{s}$ $\left(X_{j, s}\right)$ denotes the set of numbers enumerated in $X\left(X_{j}\right)$ by the end of stage $s$.

We assume the reader is familiar with Soare [9].

\section{Strongly noncappable degrees.}

Definition. (a) An r.e. degree a is non-b-cappable if

$$
\forall \mathbf{c}(\mathbf{c} \$ \mathbf{b} \rightarrow \exists \mathbf{d}(\mathbf{d} \leqslant \mathbf{a}, \mathbf{c} \text { and } \mathbf{d} \$ \mathbf{b})) ;
$$

otherwise $\mathbf{a}$ is b-cappable.

(b) An r.e. degree $\mathbf{a}$ is strongly noncappable (s.n.c.) if $\mathbf{a}>\mathbf{0}$ and $\forall \mathbf{b}<\mathbf{a}$ (a is non-b-cappable).

SNC denotes the set of strongly noncappable degrees. Instead of non-0-cappable and 0-cappable we usually say noncappable (n.c.) and cappable, respectively. A nonzero r.e. degree $\mathbf{a}$ is cappable iff it is half of a minimal pair, i.e. if there is a $\mathbf{b} \mid \mathbf{a}$ such that $\mathbf{a} \cap \mathbf{b}=\mathbf{0}$. The existence of minimal pairs was shown in Lachlan [5] and Yates [11], that of incomplete noncappable degrees in Yates [11]. It is easily shown that the n.c. degrees form a filter, i.e. for n.c. $\mathbf{a}$ and $\mathbf{b} \geqslant \mathbf{a}, \mathbf{b}$ is n.c., and for n.c. degrees $\mathbf{a}_{0}, \ldots, \mathbf{a}_{n}$ s.t. $\mathbf{a}_{0} \cap \cdots \cap \mathbf{a}_{n}$ exists, $\mathbf{a}_{0} \cap \cdots \cap \mathbf{a}_{n}$ is n.c. More facts about non-b-cappable degrees can be found in [2]. Obviously $\mathbf{0}^{\prime}$ is s.n.c. and every s.n.c. degree is n.c. There are n.c. degrees, however, which are not s.n.c. This follows from the existence of a branching n.c. degree [1, Theorem 1.1], i.e. an n.c. degree $\mathbf{a}$ s.t. $\mathbf{a}=\mathbf{a}_{0} \cap \mathbf{a}_{1}$ for r.e. degrees $\mathbf{a}_{0}, \mathbf{a}_{1}>\mathbf{a}$. Note that if $\mathbf{a}$ is s.n.c. and $\mathbf{a} \mid \mathbf{b}$, then $\mathbf{a} \cap \mathbf{b}$ does not exist. This is the crucial property of s.n.c. degrees which will be used in the following.

THEOREM 1. There is an incomplete strongly noncappable degree.

An immediate consequence of Theorem 1 is that not every nonzero incomplete r.e. degree is half of a pair of incomparable r.e. degrees which have an infimum.

Corollary 1. There is an r.e. degree $\mathbf{a} \neq \mathbf{0 ,} \mathbf{0}^{\prime}$ such that for all $\mathbf{b} \mid \mathbf{a}, \mathbf{a} \cap \mathbf{b}$ does not exist. 
Proof of TheOREM 1. By a finite injury priority argument we construct an r.e. set $A$ such that $\mathbf{a}=\operatorname{deg} A$ is incomplete and s.n.c.

To make $A$ nonrecursive and incomplete we meet the requirements

$$
\mathrm{P}_{e}: A^{(0)} \neq\{e\} \text { and } \mathrm{N}_{e}: C \neq\{e\}^{A}
$$

for a fixed nonrecursive r.e. set $C$ and all numbers $e$. These requirements are handled by standard methods. To satisfy $\mathrm{P}_{e}$ we wait for a stage $s$ and a number $x \in \omega^{(0, e)}$ s.t. $\{e\}_{s}(x)=0$. Then-provided no higher priority requirement restrains $x$ from $A$-we put $x$ into $A^{(0)}$ at stage $s+1$, thus establishing a disagreement between $A^{(0)}$ and $\{e\}$. To meet $N_{e}$ we use Sacks' preservation strategy. Given an effective enumeration $\left\langle C_{s}\right.$ : $s<\omega\rangle$ of $C$, we define

$$
l(e, s)=\max \left\{x: \forall y<x\left(C_{s}(y)=\{e\}_{s}^{A_{s}}(y)\right)\right\} \quad \text { (length function) }
$$

and

$$
r(e, s)=\max \left\{u\left(e, A_{s}, x, s\right): x \leqslant l(e, s)\right\} \quad \text { (restraint function). }
$$

By standard arguments $\mathrm{N}_{e}$ is met and $\lim _{s} r(e, s)<\omega$ exists provided that the injury set

$$
I_{e}=\left\{x: \exists s\left(x \in A_{s+1}-A_{s} \text { and } x<r(e, s)\right)\right\}
$$

is finite.

The main task of the construction is to satisfy

$$
\forall \mathbf{v}<\mathbf{a} \forall \mathbf{w} \$ \mathbf{v} \exists \mathbf{e}(\mathbf{e} \leqslant \mathbf{a}, \mathbf{w} \text { and } \mathbf{e} \$ \mathbf{v}),
$$

which, together with the nonrecursiveness of $A$, implies a is s.n.c. Since, for $\mathbf{v}$ and $\mathbf{w}$ as in (1) s.t. $\mathbf{w} \leqslant \mathbf{a}, \mathbf{w} \leqslant \mathbf{a}, \mathbf{w}$ and $\mathbf{w} \$ \mathbf{v}$, (1) may be replaced by the weaker condition

$$
\forall \mathbf{v}<\mathbf{a} \forall \mathbf{w} \neq \mathbf{a} \exists \mathbf{e}(\mathbf{e} \leqslant \mathbf{a}, \mathbf{w} \text { and } \mathbf{e} \$ \mathbf{v}) \text {. }
$$

To satisfy (2), for all $e=\left\langle e_{0}, e_{1}, e_{2}\right\rangle$ we construct r.e. sets $E_{e}$ such that

$$
\left(W_{e_{0}}=\left\{e_{1}\right\}^{A}, A \$_{T} W_{e_{0}} \text { and } W_{e_{2}} \hbar_{T} A\right) \rightarrow\left(E_{e} \leqslant_{T} A, W_{e_{2}} \text { and } E_{e} \$_{T} W_{e_{0}}\right) \text {. }
$$

The premise $W_{e_{0}}=\left\{e_{1}\right\}^{A}$ is used to control the enumeration of $W_{e_{0}}$ by controlling that of $A$. We ensure (3) by meeting the requirements

$$
\mathbf{R}_{\langle e, i\rangle}:\left(W_{e_{0}}=\left\{e_{1}\right\}^{A}, A \$_{T} W_{e_{0}} \text { and } W_{e_{2}} \hbar_{T} A\right) \rightarrow\left(E_{e} \neq\{i\}^{W_{e_{0}}}\right)
$$

and satisfying the conditions

$$
\begin{aligned}
& E_{e} \leqslant_{T} W_{e_{2}}, \\
& W_{e_{0}}=\left\{e_{1}\right\}^{A} \rightarrow E_{e} \leqslant_{T} A
\end{aligned}
$$

for all $e=\left\langle e_{0}, e_{1}, e_{2}\right\rangle$ and $i$.

The basic idea to meet requirement $\mathrm{R}_{\langle e, i\rangle}$ is that for meeting $\mathrm{P}_{e}$ : We wait for a number $x \in \omega^{(e+1, i)}$ and a stage $s$ such that $\{i\}_{s}^{W_{e, 0}, s}(x)=0$. If we then put $x$ into $A$ $\mathbf{R}_{\langle e, i\rangle}$ is met unless the computation $\{i\}_{s}^{W_{e 0 .} s}(x)$ is not $W_{e_{0}}$-correct, i.e.

$$
W_{e_{0}, s} \backslash u\left(i, W_{e_{0}, s}, x, s\right) \neq W_{e_{0}} \uparrow u\left(i, W_{e_{0}, s}, x, s\right) \text {. }
$$


To prevent $W_{e_{0}}$ from changing below $u\left(i, W_{e_{0}, s}, x, s\right)$ after stage $s$, we attack $\mathrm{R}_{\langle e, i\rangle}$ with $x$ only if

$$
W_{e_{0}, s} \backslash u\left(i, W_{e_{0}, s}, x, s\right)=\left\{e_{1}\right\}_{s}^{A_{s}} \uparrow u\left(i, W_{e_{0}, s}, x, s\right) .
$$

In this case we impose a restraint on $A$ to preserve the right side of (6). Then either the left side of (6) does not change and, therefore, $\{i\}^{W_{e_{0}}}(x)=0$, or it is not correct, which implies $W_{e_{0}} \neq\left\{e_{1}\right\}^{A}$. In either case, requirement $\mathbf{R}_{\langle e, i\rangle}$ is met.

This procedure to meet $\mathbf{R}_{\langle e, i\rangle}$ is restricted by the need to satisfy (4) and (5). Condition (4) is ensured by the permitting method: A number $x$ is allowed to enter $E_{e}$ only at such stages $s+1$ for which a $y<x$ exists such that $y \in W_{e_{2}, s+1}-W_{e_{2}, s}$. A similar action to satisfy (5), i.e. to put a number $y \leqslant x$ into $A$ whenever $x$ is put into $E_{e}$, is in conflict with the necessity to preserve the right side of (6), which requires us to restrain certain numbers from $A$. To solve this conflict, for $e$ such that $W_{e_{0}}=\left\{e_{1}\right\}^{A}$, we define an $A$-recursive function $f_{e}(x)$ and a recursive monotonic approximation $f_{e}(x, s)$ to $f_{e}(x)$ such that for sufficiently many $x$ and $s, f_{e}(x, s)$ is greater than the restraint required to preserve the right side of (6). Only for such $x$ and $s$ we will put $x$ into $E_{e, s+1}$, and in this case we enumerate a new number less than or equal to $f_{e}(x, s)$ in $A_{s+1}$. It follows that

$$
\forall x, s\left(A_{s} \backslash f_{e}(x)+1=A\left\lceil f_{e}(x)+1 \rightarrow E_{e, s} \backslash x+1=E_{e} \uparrow x+1\right),\right.
$$

whence (5) is satisfied.

To define $f_{e}$, for $e=\left\langle e_{0}, e_{1}, e_{2}\right\rangle$, we first inductively define the standard marker ( function) $\gamma$ of $A$ (with respect to $\left\langle A_{s}: s<\omega\right\rangle$ ):

$$
\gamma(x, 0)=0, \quad \gamma(x, s+1)= \begin{cases}s+1 & \text { if } A_{s+1} \uparrow x \neq A_{s} \uparrow x \\ \gamma(x, s) & \text { otherwise. }\end{cases}
$$

$\gamma(x, s)$ is recursive, nondecreasing in both arguments, and for all $x, \lim _{s} \gamma(x, s)<\omega$ exists. We denote $\lim _{s} \gamma(x, s)$ by $\gamma(x)$. Then $\gamma(x)$ is an $A$-recursive function. The crucial property of standard marker functions is stated in the following lemma.

Lemma 1. Let $A$ be an r.e. set, $\gamma$ a standard marker of $A$ with respect to some effective enumeration $\left\langle A_{n}: n<\omega\right\rangle$ of $A, B$ an infinite set, and $h$ a function such that $A \$_{T} B \oplus h$. Then there are infinitely many $x \in B$ such that $\gamma(x)>h(x)$.

Proof. Assume, for a contradiction, there are only finitely many $x \in B$ s.t. $\gamma(x)>h(x)$, say $x_{0}$ is the greatest such $x$. Then

$$
\forall x\left(x>x_{0} \text { and } x \in B \rightarrow A_{h(x)} \mid x=A \uparrow x\right) .
$$

Since $B$ is infinite this implies $A \leqslant_{T} B \oplus h$.

Based on the definition of $\gamma$ we define, for $e=\left\langle e_{0}, e_{1}, e_{2}\right\rangle$ and $x=\left\langle x_{0}, x_{1}, x_{2}\right\rangle$,

$$
f_{e}(x, s)=\left\langle x_{0}, x_{1}, x_{2}, \max \left\{u\left(e_{1}, A_{s}, y, t\right): y \leqslant \gamma(x, s) \text { and } t \leqslant s\right\}\right\rangle .
$$

The binary function $f_{e}$ is recursive and nondecreasing in both arguments. For $e$ such that $\left\{e_{1}\right\}^{A}$ is total, $\lim _{s} f_{e}(x, s)=\sup _{s} f_{e}(x, s)<\omega$ exists for all $x$. For such $e$ we set $f_{e}(x)=\lim _{s} f_{e}(x, s)$. If defined, $f_{e}(x)$ is a total $A$-recursive function. Note that $x \leqslant f_{e}(x, s)$ and for $x \in \omega^{(i, j)}, f_{e}(x, s)$ is an element of $\omega^{(i, j)}$ too. 
We now turn to the formal description of the construction. The priority ordering of the requirements is given by

$$
\mathrm{N}_{0}>\mathrm{R}_{0}>\mathrm{P}_{0}>\cdots>\mathrm{N}_{n}>\mathrm{R}_{n}>\mathrm{P}_{n}>\mathrm{N}_{n+1}>\mathrm{R}_{n+1}>\mathrm{P}_{n+1}>\cdots .
$$

For any $\langle e, i\rangle$ and $s, R(\langle e, i\rangle, s)$ denotes the restraint imposed by requirement $\mathbf{R}_{\langle e, i\rangle}$ at the end of stage $s$; it is defined at stage $s$ of the construction below. The requirement $\mathbf{R}_{\langle e, i\rangle}$, where $e=\left\langle e_{0}, e_{1}, e_{2}\right\rangle$, requires attention at stage $s+1$ if there is an $x \in \omega^{(e+1, i)}$ such that the following conditions hold:

$$
\begin{aligned}
& R(\langle e, i\rangle, s)=0, \\
& \{i\}_{s}^{W_{c \mid 1 .} \cdot(}(x)=0 \quad \text { and } \quad x \notin E_{e, s}, \\
& \gamma(x, s) \geqslant u, \quad \text { where } u=u\left(i, W_{e_{0}, s}, x, s\right), \\
& W_{e_{0}, s}\left|u=\left\{e_{1}\right\}_{s}^{A}\right| u, \\
& f_{e}(x, s) \geqslant \max (\{r(k, s): k \leqslant\langle e, i\rangle\} \cup\{R(k, s): k<\langle e, i\rangle\}),
\end{aligned}
$$

and

$$
\exists y<x\left(y \in W_{e_{2}, s+1}-W_{e_{2}, s}\right) .
$$

Requirement $\mathrm{P}_{e}$ requires attention at stage $s+1$ if

$$
\forall y \in \omega^{(0, e)}\left(\{e\}_{s}(y) \downarrow \rightarrow A_{s}(y)=\{e\}_{s}(y)\right)
$$

and there is an $x \in \omega^{(0, e)}$ such that

$$
\{e\}_{s}(x)=0 \text { and } x \geqslant \max (\{r(k, s): k \leqslant e\} \cup\{R(k, s): k \leqslant e\}) .
$$

\section{CONSTRUCTION.}

Stage 0 . For all $x$ and $e$, set $R(x, 0)=0$ and define $l(x, 0), r(x, 0), \gamma(x, 0)$, and $f_{e}(x, 0)$ as described above.

Stage $s+1$. Choose the highest priority requirement which requires attention.

Case 1. $\mathrm{P}_{e}$ is this requirement. Then choose the least $x \in \omega^{(0, e)}$ which satisfies (8.2). Put $x$ into $A$ and for all $k$ set

$$
R(k, s+1)= \begin{cases}R(k, s) & \text { if } k \leqslant e, \\ 0 & \text { if } k>e .\end{cases}
$$

Case 2. $\mathbf{R}_{\langle e, i\rangle}, e=\left\langle e_{0}, e_{1}, e_{2}\right\rangle$, is this requirement. Then choose the least $x \in \omega^{(e+1 . i)}$ for which (7.1)-(7.6) hold. Put $x$ into $E_{e}$ and $f_{c}(x, s)$ into $A$. For $k<\omega$. define

$$
R(k, s+1)= \begin{cases}R(k, s) & \text { if } k<\langle e, i\rangle, \\ \max \left\{u\left(e_{1}, A_{s}, y, s\right): y<u\left(i, W_{e_{1}, . s}, x, s\right)\right\}, & \text { if } k=\langle e, i\rangle, \\ 0 & \text { if } k>\langle e, i\rangle .\end{cases}
$$

Case 3. There is no requirement which requires attention. Then set $R(k, s+1)=$ $R(k, s)$ for all $k$.

In any case define $l(x, s+1), r(x, s+1), \gamma(x, s+1)$ and $f_{\ell}(x, s+1)$ as described above. If Case $1(2)$ holds, we say $\mathrm{P}_{e}\left(\mathrm{R}_{\left\langle u^{\prime}, i\right\rangle}\right)$ receives atttention or is active at stage $s+1$. 
This completes the construction. The construction is effective. Hence the functions $R(x, s), l(x, s), r(x, s), \gamma(x, s)$ and $f_{e}(x, s)$ are recursive, and the sets $A$ and $E_{e}$ are recursively enumerable. Note that a number $x \in \omega^{(0 . e)}$ enters $A$ only at a stage at which $\mathrm{P}_{e}$ is active; a number $x \in \omega^{(e+1, i)}$ enters $E_{e}$ or $A$ only at a stage at which $\mathrm{R}_{\langle e, i\rangle}$ is active. To verify that the constructed sets have the desired properties, we prove a series of lemmas.

LEMMA 2. (a) Every requirement requires attention at most finitely often.

(b) For all $e, \lim _{s} R(e, s)<\omega$ exists.

Proof. Part (a) is shown by induction on the priority of the requirement. Given a requirement $\mathrm{R}$, by inductive hypothesis fix a stage $s_{0}$ after which no higher priority requirement requires attention. Assume $\mathrm{R}$ requires attention at stage $s_{1}>s_{0}$. Then $\mathrm{R}$ receives attention at stage $s_{1}$. If $\mathrm{R}$ is requirement $\mathrm{P}_{e}$, a number $x \in \omega^{(0, e)}$ such that $\{e\}_{s_{1}}(x)=0$ is put into $A$ at stage $s_{1}$, whence by $(8.1) \mathrm{P}_{e}$ does not require attention after stage $s_{1}$. If $\mathrm{R}$ is requirement $\mathrm{R}_{\langle e, i\rangle}$, then $R\left(\langle e, i\rangle, s_{1}\right)>0$, and by choice of $s_{0}$ and $s_{1}>s_{0}, R(\langle e, i\rangle, s)=R\left(\langle e, i\rangle, s_{1}\right)$ for all $s \geqslant s_{1}$. Hence by (7.1), $\mathrm{R}_{\langle e . i\rangle}$ does not require attention after stage $s_{1}$. This completes the proof of (a). Part (b) follows from (a), since $R(e, s+1) \neq R(e, s)$ implies $\mathrm{R}_{e}$ or a higher priority requirement is active at stage $s+1$.

Lemma 3. For every e, $\mathrm{N}_{e}$ is met and $\lim _{s} r(e, s)<\omega$ exists.

Proof. For any $e$ and $s$, a number $x<r(e, s)$ can enter $A$ at stage $s+1$ only for the sake of a requirement $\mathrm{P}_{k}$ or $\mathrm{R}_{k}$ where $k<e$. Hence by Lemma 2 the injury set $I_{e}$ is finite. As in Soare [9, Lemmas 1.1 and 1.2] we may conclude Lemma 3 holds.

We set $r(e)=\sup _{s} r(e, s)$ and $R(e)=\lim _{s} R(e, s)$.

Lemma 4. For all e, $\mathrm{P}_{e}$ is met.

Proof. For a contradiction assume $A^{(0)}=\{e\}$. Then $\mathrm{P}_{e}$ never receives attention, since if $\mathrm{P}_{e}$ is active at stage $s+1$ there is an $x \in \omega^{(0, e)}$ such that

$$
1=A^{(0)}(x)=A_{s+1}^{(0)}(x) \neq\{e\}_{s}(x)=\{e\}(x)=0 .
$$

Since a number $x \in \omega^{(0, e)}$ can enter $A$ only if $\mathrm{P}_{e}$ is active, it follows that no $x \in \omega^{(0, e)}$ is in $A$, i.e. by assumption

$$
\forall x \in \omega^{(0, e)}(A(x)=\{e\}(x)=0) .
$$

This implies that (8.1) holds for all $s$. By Lemmas 2 and 3, choose $s_{0}$ and $x_{0} \in \omega^{(0, e)}$ such that no requirement of higher priority than $\mathrm{P}_{e}$ requires attention after stage $s_{0}$ and such that

$$
\forall s \forall k \leqslant e\left(r(k) \leqslant x_{0} \text { and } R(k, s) \leqslant x_{0}\right) .
$$

By (9) there is a stage $s_{1}>s_{0}$ such that $\{e\}_{s_{1}}\left(x_{0}\right)=0$, so $\mathrm{P}_{e}$ requires and receives attention at stage $s_{1}+1$, contradiction.

Lemma 5. For all e and $i, \mathrm{R}_{\langle e, i\rangle}$ is met. 
Proof. Fix $e=\left\langle e_{0}, e_{1}, e_{2}\right\rangle$ and $i$, and for a contradiction assume $\mathbf{R}_{\langle e, i\rangle}$ is not met, i.e.

$$
\begin{aligned}
& W_{e_{0}}=\left\{e_{1}\right\}^{A}, \\
& A \$_{T} W_{e_{0}}, \\
& W_{e_{2}} \$_{T} A, \\
& E_{e}=\{i\}^{W_{e_{0}} .}
\end{aligned}
$$

We distinguish the following two cases.

Case 1. $R(\langle e, i\rangle)>0$. Choose the least $s$ such that

$$
\forall t>s(R(\langle e, i\rangle, t)>0) \text {. }
$$

Then $\mathbf{R}_{\langle e, i\rangle}$ is active at stage $s+1$, i.e. there is an $x \in \omega^{(e+1, i)}$ which satisfies (7.1)-(7.6) and which is put into $E_{e}$ at stage $s+1$. Moreover,

$$
R(\langle e, i\rangle, s+1)=\max \left\{u\left(e_{1}, A_{s}, y, s\right): y<u\left(i, W_{e_{0}, s}, x, s\right)\right\} .
$$

By (14) neither $\mathbf{R}_{\langle e, i\rangle}$ nor a requirement of higher priority is active after stage $s+1$. Hence

$$
\forall t>s(R(\langle e, i\rangle, t)=R(\langle e, i\rangle, s+1))
$$

and no number less than $R(\langle e, i\rangle, s+1)$ enters $A$ after stage $s+1$. Since at stage $s+1$ only $f_{e}(x, s)$ enters $A$, which by (7.3) is greater than or equal to $R(\langle e, i\rangle, s+1)$, it follows that

$$
A_{s} \backslash R(\langle e, i\rangle, s+1)=A \uparrow R(\langle e, i\rangle, s+1) .
$$

From (7.4), (15) and (16) we can deduce that for $u=u\left(i, W_{e_{0}, s}, x, s\right)$,

$$
W_{e_{0}, s} \uparrow u=\left\{e_{1}\right\}_{s}^{A_{s}} \uparrow u=\left\{e_{1}\right\}^{A} \uparrow u .
$$

Hence by (10), $W_{e_{0}} \backslash u=W_{e_{0}, s} \backslash u$ and, therefore, by (7.2),

$$
\{i\}^{W_{e_{0}}}(x)=\{i\}_{s}^{W_{e_{0} . s}}(x)=0 .
$$

Since $x \in E_{e}$ this implies $\mathrm{R}_{\langle e, i\rangle}$ is met, contrary to our assumption.

Case 2. $R(\langle e, i\rangle)=0$. Then by Lemmas 2 and 3 we can choose $s_{0}$ and $x_{0}$ such that $\mathbf{R}_{\langle e, i\rangle}$ does not require attention after stage $s_{0}$,

$$
\begin{aligned}
& \forall s \geqslant s_{0}(R(\langle e, i\rangle, s)=0), \\
& \forall k \geqslant\langle e, i\rangle \forall s\left(r(k) \leqslant x_{0} \text { and } R(k, s) \leqslant x_{0}\right),
\end{aligned}
$$

and no number greater than $x_{0}$ is put into $E_{e}$ for the sake of requirement $\mathrm{R}_{\langle e, i\rangle}$, i.e.

$$
\forall x \in \omega^{(e+1, i)}\left(x>x_{0} \rightarrow E_{e}(x)=0\right)
$$

and, consequently, by (13),

$$
\forall x \in \omega^{(e+1, i)}\left(x>x_{0} \rightarrow E_{e}(x)=\{i\}^{W_{e_{0}}}(x)=0\right) .
$$

Since by (13) $u\left(i, W_{e_{0}}, x\right)$ is a total $W_{e_{0}}$-recursive function, it follows from (11) and Lemma 1 that the set

$$
G=\left\{x \in \omega^{(e+1, i)}: x>x_{0} \text { and } \gamma(x)>u\left(i, W_{e_{0}}, x\right)\right\}
$$


is infinite. Obviously,

$$
G \leqslant_{T} \lambda x(\gamma(x)) \oplus \lambda x\left(u\left(i, W_{e_{0}}, x\right)\right) \leqslant_{T} A \oplus W_{e_{0}},
$$

i.e. by (10), $G \leqslant_{T} A$. For $x \in G$,

$\{i\}_{s}^{W_{e_{0}, s}}(x)=0$ via a $W_{e_{0}}$-correct computation, i.e.

$$
W_{e_{0}, s} \uparrow u\left(i, W_{e_{0}, s}, x, s\right)=W_{e_{0}} \uparrow u\left(i, W_{e_{0}, s}, x, s\right),
$$$$
\gamma(x, s)>u\left(i, W_{e_{0}, s}, x, s\right), \quad \text { and }
$$$$
W_{e_{0}, s} \uparrow u\left(i, W_{e_{0}, s}, x, s\right)=\left\{e_{1}\right\}_{s}^{A_{s}} \uparrow u\left(i, W_{e_{0}, s}, x, s\right) \text { via }
$$

$A$-correct computations, i.e. $A_{s} \uparrow v=A \uparrow v$, where

$$
v=\max \left\{u\left(e_{1}, A_{s}, y, s\right): y<u\left(i, W_{e_{0}, s}, x, s\right)\right\}
$$

are true for almost all $s$. Moreover, the function

$$
s(x)= \begin{cases}\mu s>s_{0}(s \text { satisfies }(19.1)-(19.3)) & \text { if } x \in G, \\ 0 & \text { otherwise }\end{cases}
$$

is $A$-recursive and for all $x \in G$ and $s \geqslant s(x),(19.1)-(19.3)$ hold. Together with (17) and (18) this implies that for all $x \in G$ and $s \geqslant s(x)$, conditions (7.1)-(7.5) are satisfied. Since $\mathrm{R}_{\langle e, i\rangle}$ does not require attention after stage $s_{0}$, for no such $x$ and $s$, (7.6) can hold, i.e.

$$
\forall x \in G\left(W_{e_{2}, s(x)} \uparrow x=W_{e_{2}} \uparrow x\right) .
$$

Since $G$ is infinite, and $G$ and $\lambda x(s(x))$ are recursive in $A$, this implies that $W_{e_{2}}$ is recursive in $A$, a contradiction to (12).

Lemma 6. For all $e=\left\langle e_{0}, e_{1}, e_{2}\right\rangle$,

(a) $E_{e} \leqslant{ }_{T} W_{e_{2}}$,

(b) $W_{e_{0}}=\left\{e_{1}\right\}^{A} \rightarrow E_{e} \leqslant_{T} A$.

Proof. (a) By (7.6) a number is put into $E_{e}$ at stage $s+1$ only if there is a smaller number in $W_{e_{2}, s+1}-W_{e_{2}, s}$. Hence

$$
\forall s, x\left(W_{e_{2}, s} \uparrow x=W_{e_{2}} \uparrow x \rightarrow E_{e, s} \uparrow x=E_{e} \uparrow x\right),
$$

which implies $E_{e} \leqslant{ }_{T} W_{e_{2}}$.

(b) Assume $W_{e_{0}}=\left\{e_{1}\right\}^{A}$. Then $\left\{e_{1}\right\}^{A}$ is total and thus the unary function $f_{e}$ is total and $A$-recursive, as we have pointed out above. Since for all $e, e^{\prime}, x, y, s$ and $t$, $x \neq y \rightarrow f_{e}(x, s) \neq f_{e^{\prime}}(y, t)$ and $E_{e} \subseteq \omega^{(e+1)}$, it follows from the construction that

$$
\forall x \in \omega^{(e+1)} \forall s\left(x \in E_{e, s+1}-E_{e, s} \rightarrow f_{e}(x, s) \in A_{s+1}-A_{s}\right) .
$$

Hence

$$
\forall x, s\left(A_{s} \uparrow f_{e}(x)+1=A \uparrow f_{e}(x)+1 \rightarrow E_{e, s} \uparrow x+1=E_{e} \uparrow x+1\right),
$$

which implies $E_{e} \leqslant{ }_{T} A$.

As shown above, Lemmas $3-6$ imply $\operatorname{deg} A$ is s.n.c. and incomplete, which completes the proof of Theorem 1 . 
The proof of Theorem 1 actually shows that for any $\mathbf{c}>\mathbf{0}$ there is an s.n.c. degree $\mathbf{a} \neq \mathbf{c}$. Moreover, the constructed set is low (see Soare [9, Remarks 4.4 and 4.5]).

We now combine the splitting technique with the s.n.c. degree construction to show that for every $\mathbf{c} \neq \mathbf{0}, \mathbf{0}^{\prime}$ there is a (low) s.n.c. degree incomparable with $\mathbf{c}$.

THEOREM 2. Let $\mathbf{c}>\mathbf{0}$ be given. Then there are low s.n.c. degrees $\mathbf{a}_{0}$ and $\mathbf{a}_{1}$ such that $\mathbf{c} \$ \mathbf{a}_{0}, \mathbf{a}_{1}$ and $\mathbf{a}_{0} \cup \mathbf{a}_{1}=\mathbf{0}^{\prime}$.

Corollary 2. For every $\mathbf{c} \neq \mathbf{0}, \mathbf{0}^{\prime}$ there is a low s.n.c. degree incomparable with $\mathbf{c}$.

Proof. Given $\mathbf{c} \neq \mathbf{0}, \mathbf{0}^{\prime}$, by Theorem 2 split $\mathbf{0}^{\prime}$ into low s.n.c. degrees $\mathbf{a}_{0}$ and $\mathbf{a}_{1}$ s.t. $\mathbf{c} \$ \mathbf{a}_{0}, \mathbf{a}_{1}$. Then $\mathbf{c} \mid \mathbf{a}_{0}$ or $\mathbf{c} \mid \mathbf{a}_{1}$.

Corollary 3. For every r.e. degree $\mathbf{c} \neq \mathbf{0}, \mathbf{0}^{\prime}$ there is an r.e. degree a such that $\mathbf{c} \cap$ a does not exist.

Proof of Theorem 2. We combine the proof of Theorem 1 with that of the splitting theorem (see, e.g., Soare [9, Theorem 1.2]).

Fix $\mathbf{c}>\mathbf{0}, C \in \mathbf{c}$, r.e. and an effective enumeration $\left\langle C_{s}: s<\omega\right\rangle$ of $C$, and choose a complete set $A \subseteq \omega^{(0)}$ and a recursive function $g$ which enumerates $A$ without repetitions. We construct r.e. sets $A_{0}$ and $A_{1}$ such that $\mathbf{a}_{0}=\operatorname{deg} A_{0}$ and $\mathbf{a}_{1}=\operatorname{deg} A_{1}$ satisfy the theorem.

To ensure $\mathbf{a}_{0} \cup \mathbf{a}_{1}=\mathbf{0}^{\prime}$, at stage $s+1$ of the construction we put $g(s)$ into $A_{0}$ or $A_{1}$ and no other numbers are enumerated in $A_{0}^{(0)}$ and $A_{1}^{(0)}$. Then $A=A_{0}^{(0)} \cup A_{1}^{(0)}$, which implies $A \leqslant_{T} A_{0} \oplus A_{1}$.

As in the preceding proof we use the preservation strategy to make $C$ nonrecursive in $A_{0}$ and $A_{1}$. For all $e$ and for $j \leqslant 1$ we meet the requirements

$$
\mathrm{N}_{2 e+j}: C \neq\{e\}^{A_{j}} .
$$

The length function $l(2 e+j, s)$, the restraint function $r(2 e+j, s)$ and the injury set $I_{2 e+j}$ are obtained from the definitions of $l(e, s), r(e, s)$ and $I_{e}$ in the proof of Theorem 1 by replacing $A$ by $A_{j}$.

To make $\mathbf{a}_{0}$ and $\mathbf{a}_{1}$ s.n.c. it suffices to construct r.e. sets $E_{e}^{j}$ such that for all $e=\left\langle e_{0}, e_{1}, e_{2}\right\rangle, i<\omega$ and $j \leqslant 1$, the requirements

$$
\mathrm{R}_{\langle 2 e+j, i\rangle}:\left(W_{e_{0}}=\left\{e_{1}\right\}^{A_{j}}, A_{j} \$_{T} W_{e_{0}} \text { and } W_{e_{2}}{ }_{T} A_{j}\right) \rightarrow E_{j}^{e} \neq\{i\}^{W_{e_{0}}},
$$

and conditions

$$
\begin{aligned}
& E_{e}^{j} \leqslant_{T} W_{e_{2}} \\
& W_{e_{0}}=\left\{e_{1}\right\}^{A_{j}} \rightarrow E_{e}^{j} \leqslant_{T} A_{j}
\end{aligned}
$$

are satisfied.

Let $\gamma_{j}$ be the standard marker of $A_{j}$ with respect to $\left\langle A_{j, s}: s\langle\omega\rangle\right.$, and define the functions $f_{e}^{j}$ by replacing $A$ and $\gamma$ by $A_{j}$ and $\gamma_{j}$, respectively, in the definition of $f_{e}$ in the foregoing proof $(j=0,1)$.

The requirement $\mathrm{R}_{\langle 2 e+j, i\rangle}$, where $e=\left\langle e_{0}, e_{1}, e_{2}\right\rangle$, requires attention at stage $s+1$ if there is an $x \in \omega^{(2 e+j+1, i)}$ such that

$$
R(\langle 2 e+j, i\rangle, s)=0,
$$




$$
\begin{aligned}
& \{i\}_{s}^{W_{c_{0} .} s}(x)=0, \\
& \gamma_{j}(x, s) \geqslant u, \quad \text { where } u=u\left(i, W_{e_{0} . s}, x, s\right), \\
& W_{e_{0}, s}\left|u=\left\{e_{1}\right\}_{s}^{A, .1}\right| u, \\
& f_{e}^{j}(x, s) \geqslant \max (\{r(k, s): k \leqslant\langle 2 e+j, i\rangle\} \\
& \quad \cup\{R(k, s): k<\langle 2 e+j, i\rangle\}), \text { and } \\
& \exists y<x\left(y \in W_{e_{2} . s+1}-W_{e_{2} . s}\right) .
\end{aligned}
$$

We say $k$ needs protection at stage $s+1$ if $g(s)<\max \{r(k, s), R(k, s)\}$.

Construction.

Stage 0 . For $x, e<\omega$ and $j \leqslant 1$, set $R(x, 0)=0$ and define $l(x, 0), r(x, 0), \gamma_{j}(x, 0)$ and $f_{e}^{j}(x, 0)$ as described above.

Stage $s+1$. If there is a number $y$ such that $y$ needs protection or $\mathbf{R}_{y}$ requires attention at stage $s+1$, then let $\langle 2 e+j, i\rangle$, where $e=\left\langle e_{0}, e_{1}, e_{2}\right\rangle$ and $j \leqslant 1$, be the least such number and distinguish the two cases below. Otherwise put $g(s)$ into $A_{0}$ and set $R(k, s+1)=R(k, s)$ for all $k$.

Case 1. $\mathbf{R}_{\langle 2 e+j, i\rangle}$ requires attention. Then choose the least $x \in \omega^{(2 e+j+1, i)}$ which satisfies (22.1)-(22.6). Put $x$ into $E_{e}^{j}, f_{e}^{j}(x, s)$ into $A_{j}$, and $g(s)$ into $A_{1-j}$. Define

$$
R(k, s+1)= \begin{cases}R(k, s) & \text { if } k<\langle 2 e+j, i\rangle, \\ \max \left\{u\left(e_{1}, A_{j, s}, y, s\right): y<u\left(i, W_{e_{0} . s}, x, s\right)\right\} & \text { if } k=\langle 2 e+j, i\rangle, \\ 0 & \text { if } k>\langle 2 e+j, i\rangle .\end{cases}
$$

Case 2. Otherwise. Then put $g(s)$ into $A_{1-j}$ and define

$$
R(k, s+1)= \begin{cases}R(k, s) & \text { if } k \leqslant\langle 2 e+j, i\rangle, \\ 0 & \text { if } k>\langle 2 e+j, i\rangle .\end{cases}
$$

In any case, define $l(x, s+1), r(x, s+1), \gamma_{j}(x, s)$ and $f_{e}^{j}(x, s)$ for all $x, e<\omega$ and $j \leqslant 1$ as described above.

Obviously the construction is effective and $A=A_{0}^{(0)} \cup A_{1}^{(0)}$. The latter implies $\mathbf{a}_{0} \cup \mathbf{a}_{1}=\mathbf{0}^{\prime}$. To verify that $\mathbf{c} \$ \mathbf{a}_{0}, \mathbf{a}_{1}$ and $\mathbf{a}_{0}, \mathbf{a}_{1}$ are low and s.n.c., we first prove

LEMMA 7. For all numbers $k$ the following hold:

(i) The injury set $I_{k}$ is finite.

(ii) Requirement $\mathrm{N}_{k}$ is met.

(iii) $\lim _{s} l(k, s)$ and $\lim _{s} r(k, s)$ exist and are finite.

(iv) Requirement $\mathrm{R}_{k}$ requires attention at most finitely often.

(v) $\lim _{s} R(k, s)$ exists and is finite.

(vi) $k$ needs protection at at most finitely many stages.

The claims of Lemma 7 are proven in the given order by a simultaneous induction. The inductive step is like the proofs of Lemmas 2 and 3. For the proof of (i) and (iv) we have only to note that for $k=\langle 2 e+j, i\rangle, j \leqslant 1$, there is a stage $s_{0}$ such that for all $s>s_{0}$,

$$
g(s) \notin I_{k} \text {, i.e. } g(s)<r(k, s) \rightarrow g(s) \notin A_{j . s+1}
$$


and

$$
g(s)<R(k, s) \rightarrow g(s) \notin A_{j . s+1} .
$$

By construction, a stage $s_{0}$ has this property if after stage $s_{0}$ for no $k^{\prime}<k, \mathrm{R}_{k^{\prime}}$ requires attention or $k^{\prime}$ needs protection, and by inductive hypotheses (iv) and (vi) such an $s_{0}$ exists.

(vi) is an immediate consequence of (iii) and (v).

Lemma 7(ii) implies $\mathbf{c} \$ \mathbf{a}_{0}, \mathbf{a}_{1}$, and from Lemma 7(iii) we can conclude, as in Soare [9, Remarks 4.4 and 4.5], that $\mathbf{a}_{0}$ and $\mathbf{a}_{1}$ are low. Finally, that the requirements $\mathrm{R}_{\langle 2 e+j, i\rangle}$ and conditions (20) and (21) are satisfied for all $e=\left\langle e_{0}, e_{1}, e_{2}\right\rangle, i<\omega$ and $j \leqslant 1$ follows from Lemma 7 as Lemmas 5 and 6 follow from Lemmas 2 and 3 in the proof of Theorem 1.

This completes the proof of Theorem 2.

The proof of Theorem 2 can be combined with a technique of Robinson [8] for handling low oracle sets to do the construction of $\mathbf{a}_{0}$ and $\mathbf{a}_{1}$ above any low r.e. degree $\mathbf{d} \neq \mathbf{c}$. This implies

$$
\forall \mathbf{d} \text { low } \forall \mathbf{c} \$ \mathbf{d} \exists \mathbf{a} \text { low (a is s.n.c., } \mathbf{d}<\mathbf{a} \text { and } \mathbf{c} \$ \mathbf{a}) .
$$

An easy consequence of (23) is that the s.n.c. degrees form an automorphism basis, i.e. any two order automorphisms of $\mathbf{R}$ which agree on $\mathbf{S N C}$ must be identical. The s.n.c. degrees do not generate $\mathbf{R}$ (under $\cup$ and $\cap$ ), however, since they are contained in the proper filter of noncappable degrees.

The set SNC does not have interesting closure properties. Trivially, SNC is closed under finite infima (if they exist), since a finite set of s.n.c. degrees has an infimum iff it has a least element. SNC is not closed downwards in $\mathbf{R}$ or $\mathbf{R}-\{\mathbf{0}\}$, however, since every s.n.c. degree is n.c., and every n.c. degree bounds a nonzero cappable degree. That SNC is not closed under finite suprema, and therefore is not closed upwards, can be shown as follows: The proof of Theorem 2 can be combined with that of Lachlan's nondiamond theorem [5, Theorem 5] to prove

$$
\begin{array}{r}
\forall \mathbf{a}_{0}, \mathbf{a}_{1}\left(\mathbf{a}_{0} \cup \mathbf{a}_{1}=\mathbf{0}^{\prime} \rightarrow \exists i \leqslant 1 \exists \mathbf{a}_{i 0}, \mathbf{a}_{i 1}\right. \\
\left.\left(\mathbf{a}_{i}=\mathbf{a}_{i 0} \cup \mathbf{a}_{i 1} \text { and } \mathbf{a}_{i 0} \text { and } \mathbf{a}_{i 1} \text { are s.n.c. }\right)\right) .
\end{array}
$$

Lachlan [7] and, independently, Shoenfield and Soare (unpublished) have shown that there are incomparable r.e. degrees $\mathbf{a}_{0}$ and $\mathbf{a}_{1}$ s.t. $\mathbf{a}_{0} \cup \mathbf{a}_{1}=\mathbf{0}^{\prime}$ and $\mathbf{a}_{0} \cap \mathbf{a}_{1}$ exists. Since such degrees $\mathbf{a}_{0}$ and $\mathbf{a}_{1}$ are not s.n.c., an application of (24) yields a pair of s.n.c. degrees whose supremum is not strongly noncappable.

Obviously not every nonzero r.e. degree can be split into two s.n.c. degrees. Examples of degrees which allow such a splitting are given in Theorem 2 and in (24). We can obtain more examples by combining the s.n.c. construction with the low nondiamond technique of [2]: If $\mathbf{a}$ is low cuppable, i.e. if there is a low b s.t. $\mathbf{a} \cup \mathbf{b}=\mathbf{0}^{\prime}$, then a can be split into two s.n.c. degrees. ${ }^{2}$

\footnotetext{
${ }^{2}$ Recently Ambos-Spies, Jockusch, Shore and Soare have shown that every n.c. degree is low cuppable. Hence an r.e. degree can be split into s.n.c. degrees iff it is noncappable.
} 
All constructions mentioned above are finite (or no-) injury arguments and automatically yield low degrees. We do not know whether there are s.n.c. degrees which are not low.

We conclude this section with an application of the above results to ascending sequences of uniformly r.e. degrees. We need the following notions: A sequence $\left\langle\mathbf{c}_{n}\right.$ : $n\langle\omega\rangle$ of r.e. degrees is ascending if $\forall n\left(\mathbf{c}_{n} \leqslant \mathbf{c}_{n+1}\right)$ and $\forall n \exists m\left(\mathbf{c}_{m} \$ \mathbf{c}_{n}\right)$; it is uniformly recursively enumerable (u.r.e.) if there is a recursive function $f$ s.t. $\forall n\left(W_{f(n)} \in \mathbf{c}_{n}\right)$. A pair of incomparable r.e. degrees $\mathbf{a}$ and $\mathbf{b}$ is an exact pair for $\left\langle\mathbf{c}_{n}\right.$ : $n<\omega\rangle$ if $\forall n\left(\mathbf{c}_{n}<\mathbf{a}, \mathbf{b}\right)$ and $\forall \mathbf{d}<\mathbf{a}, \mathbf{b} \exists n\left(\mathbf{d}<\mathbf{c}_{n}\right)$. An exact pair $(\mathbf{a}, \mathbf{b})$ of $\left\langle\mathbf{c}_{n}: n<\omega\right\rangle$ is minimal if $\mathbf{a}$ and $\mathbf{b}$ are minimal upper bounds for $\left\langle\mathbf{c}_{n}: n\langle\omega\rangle\right.$. An upper bound $\mathbf{a}$ of $\left\langle\mathbf{c}_{n}: n\langle\omega\rangle\right.$ is uniform if there is an r.e. set $A \in \mathbf{a}$ and recursive functions $f$ and $g$ such that $\forall n\left(W_{f(n)} \in \mathbf{c}_{n}\right.$ and $\left.W_{f(n)}=\{g(n)\}^{A}\right)$.

Cooper [3] has shown that there are u.r.e. ascending sequences with minimal upper bounds, while Sacks (see, e.g., Soare [9, Theorem 3.1]) has shown that no u.r.e. ascending sequence has a least upper bound and uniform upper bounds are not minimal. Yates [11] constructed a u.r.e. ascending sequence with exact pair; this exact pair consists of uniform upper bounds, i.e. it is not minimal. In [2] we have shown there exist u.r.e. ascending sequences with minimal exact pairs, and in [1] that there exist such sequences without exact pairs.

Note that for an exact pair $(\mathbf{a}, \mathbf{b})$ for an ascending sequence, $\mathbf{a} \cap \mathbf{b}$ does not exist. Conversely any pair of r.e. degrees without infimum is an exact pair for an ascending sequence of r.e. degrees. That in certain cases this sequence can be chosen to be u.r.e. is an easy consequence of Yates [12, Theorem 8].

LeMma 8. If $\mathbf{a}$ and $\mathbf{b}$ are $\operatorname{low}_{2}$ and $\mathbf{a} \cap \mathbf{b}$ does not exist, then $(\mathbf{a}, \mathbf{b})$ is an exact pair for a u.r.e. ascending sequence.

For a proof of Lemma 8, see [2, Corollary 5].

Lemma 9. If $\mathbf{a}$ and $\mathbf{b}$ are $\operatorname{low}_{2}, \mathbf{a} \mid \mathbf{b}$ and $\mathbf{a}$ is s.n.c., then there is a u.r.e. ascending sequence for which $(\mathbf{a}, \mathbf{b})$ is an exact pair and a a minimal upper bound.

Proof. By Lemma 8 and the definition of an s.n.c. degree. (Note that if $\mathbf{a}$ is s.n.c. and $\mathbf{a} \mid \mathbf{b}$, then $\mathbf{a}$ is a minimal upper bound for $\{\mathbf{c}: \mathbf{c} \leqslant \mathbf{a}, \mathbf{b}\}$.)

COROllary 4. (a) (Ambos-SpIes [2]) There is a u.r.e. ascending sequence with a minimal exact pair.

(b) (COOPER [3]) There is a u.r.e. ascending sequence with a minimal upper bound.

Proof. (a) By Theorem 2 and Lemma 9. (b) By (a).

In the next section we obtain extensions of Corollary 4.

2. Pairs of r.e. degrees without infimum. From the s.n.c. degree construction we can extract the construction of a pair of r.e. degrees $\mathbf{a}$ and $\mathbf{b}$ without infimum: Using Friedberg-Muchnik type requirements we construct Turing incomparable r.e. sets $A$ and $B, \operatorname{deg} A=\mathbf{a}$ and $\operatorname{deg} B=\mathbf{b}$. In addition, for these sets we satisfy the requirements $\mathrm{R}_{\langle e, i\rangle}$ and conditions (4) and (5) of the proof of Theorem 1, where $W_{e_{2}}$ is replaced by $B$. Then $\mathbf{a}$ is a minimal upper bound of $\{\mathbf{x}: \mathbf{x} \leqslant \mathbf{a}, \mathbf{b}\}$. Since $\mathbf{a} \mid \mathbf{b}$ this 
implies $\mathbf{a} \cap \mathbf{b}$ does not exist. The strategy to meet $\mathbf{R}_{\langle e, i\rangle}$ is essentially that of the proof of Theorem 1; only condition (7.6) in the definition of requiring attention has to be replaced by

$$
x \geqslant \max (\{r(k, s): k \leqslant\langle e, i\rangle\} \cup\{R(k, s): k<\langle e, i\rangle\}),
$$

where $r(k, s)$ is the restraint imposed by the $k$ th Friedberg-Muchnik requirement at the end of stage $s$. Further, if $x$ enters $E_{e}$ at some stage, then $x$ is put into $B$ at the same stage.

We use this construction to show that every nonzero r.e. degree can be split into a pair of r.e. degrees without infimum and the pairs without infimum are dense in the r.e. degrees.

Theorem 3. Let $\mathbf{a}$ and $\mathbf{c}$ be nonzero r.e. degrees. Then there are low r.e. degrees $\mathbf{a}_{0}$ and $\mathbf{a}_{1}$ such that $\mathbf{a}=\mathbf{a}_{0} \cup \mathbf{a}_{1}, \mathbf{c} \$ \mathbf{a}_{0}, \mathbf{a}_{1}$ and $\mathbf{a}_{0} \cap \mathbf{a}_{1}$ does not exist.

Proof. W.l.o.g. we may assume $\mathbf{c} \leqslant \mathbf{a}$. Otherwise $\mathbf{c} \$ \mathbf{a}_{0}, \mathbf{a}_{1}$ follows from $\mathbf{a}=\mathbf{a}_{0}$ $\cup \mathbf{a}_{1}$, and for the proof we may replace $\mathbf{c}$ by $\mathbf{a}$.

The construction of r.e. sets $A_{0} \in \mathbf{a}_{0}$ and $A_{1} \in \mathbf{a}_{1}$ and subsidiary sets $E_{e}^{j}$ is essentially the one given in the proof of Theorem 2 . It suffices to make the following changes.

Now $A$ is an r.e. set of degree $\mathrm{a}$, and the requirement $\mathrm{R}_{\langle 2 e+j . i\rangle}, e=\left\langle e_{0}, e_{1}, e_{2}\right\rangle$ and $j \leqslant 1$, says

$$
\mathrm{R}_{\langle 2 e+j, i\rangle}:\left(W_{e_{0}}=\left\{e_{1}\right\}^{A_{j}} \text { and } A_{j} \$_{T} W_{e_{0}}\right) \rightarrow E_{e}^{j} \neq\{i\}^{W_{e_{0}}}
$$

(it would suffice to meet $\mathrm{R}_{\langle 2 e+j, i\rangle}$ for $e=\left\langle e_{0}, e_{1}, 0\right\rangle$ and $j=0$ ). Instead of (20) read

$$
E_{e}^{j} \leqslant A_{1-j}
$$

and in the definition of requiring attention replace (22.6) by

$$
g(s) \leqslant x \text {. }
$$

To verify that the construction changed in such a manner has the desired properties, we first show

LemMA 10. $\mathbf{a}=\mathbf{a}_{0} \cup \mathbf{a}_{1}$.

Proof. That $\mathbf{a} \leqslant \mathbf{a}_{0} \cup \mathbf{a}_{1}$ follows from $A=A_{0}^{(0)} \cup A_{1}^{(0)}$ as in the proof of Theorem 2. Since at stage $s+1$, by $\left(22.6^{\prime}\right)$ and $f_{e}^{j}(x, s) \geqslant x$, only numbers greater than or equal to $g(s)$ can enter $A_{0}$ and $A_{1}$, we have

$$
\forall x, s \forall j \leqslant 1\left(A_{s} \uparrow x=A \uparrow x \rightarrow A_{j, s+1} \uparrow x=A_{j} \uparrow x\right),
$$

where $A_{s}=\{g(0), \ldots, g(s)\}$. This implies $\mathbf{a}_{0} \cup \mathbf{a}_{1} \leqslant \mathbf{a}$.

Lemma 7 holds by the original proof. Hence $\mathbf{a}_{0}$ and $\mathbf{a}_{1}$ are low and $\mathbf{c} \$ \mathbf{a}_{0}, \mathbf{a}_{1}$. Since by assumption $\mathbf{c} \leqslant \mathbf{a}$, the latter and Lemma 10 imply

$$
\mathbf{a}_{0} \mid \mathbf{a}_{1} \text {. }
$$

As in the proof of Theorem 2, from Lemma 7 we can deduce that the requirements $\mathbf{R}_{\langle 2 e+j, i\rangle}$ are met: We must only replace the $W_{e_{2}}$ of the original proof by $A_{1-j}$. Then the premise $W_{e_{2}} \$_{T} A_{j}$ in the original requirements is satisfied by (25). The 
assumption that $\mathrm{R}_{\langle 2 e+j, i\rangle}$ is not met and $R(\langle 2 e+j, i\rangle)=0$ (see the proof of Lemma 5, Case 2) now implies $A \leqslant_{T} A_{j}$, contrary to Lemma 10 and (25).

That conditions $\left(20^{\prime}\right)$ and $(21)$ hold is proven as Lemma 6. For the proof of $\left(20^{\prime}\right)$ note that if $x$ is put into $E_{j}^{e}$ at stage $s+1$, then $g(s)$ is put into $A_{1-j}$. Hence, by (22.6'),

$$
\forall x, s \exists y \leqslant x\left(x \in E_{e, s+1}^{j}-E_{e, s}^{j} \rightarrow y \in A_{1-j, s+1}-A_{1-j, s}\right) .
$$

The requirements $\mathbf{R}_{\langle 2 e+j, i\rangle}$ and conditions $\left(20^{\prime}\right)$ and (21) imply $\mathbf{a}_{0}$ and $\mathbf{a}_{1}$ are both minimal upper bounds for the set $\left\{\mathbf{x}: \mathbf{x} \leqslant \mathbf{a}_{0}, \mathbf{a}_{1}\right\}$, i.e.

$$
\forall j \leqslant 1 \forall \mathbf{d}<\mathbf{a}_{j} \exists \mathbf{c} \leqslant \mathbf{a}_{0}, \mathbf{a}_{1}(\mathbf{c} \leqslant \mathbf{d}) .
$$

(25) and (26) imply $\mathbf{a}_{0} \cap \mathbf{a}_{1}$ does not exist.

In the above proof we have actually shown that any nonzero r.e. degree a can be split into incomparable low r.e. degrees $\mathbf{a}_{0}$ and $\mathbf{a}_{1}$ such that $\mathbf{a}_{0}$ and $\mathbf{a}_{1}$ are minimal upper bounds of $\left\{\mathbf{x}: \mathbf{x} \leqslant \mathbf{a}_{0}, \mathbf{a}_{1}\right\}$. Together with Lemma 8 this implies

COROllary 5. Every nonzero r.e. degree can be split into a minimal exact pair for some u.r.e. ascending sequence.

Lachlan [7] has shown the correspondng result to Theorem 3 for pais of r.e. degrees with infimum, namely that every nonzero r.e. degree a can be split into incomparable r.e. degrees $\mathbf{a}_{0}$ and $\mathbf{a}_{1}$ such that $\mathbf{a}_{0} \cap \mathbf{a}_{1}$ exists.

Using a technique of Robinson [8], the construction for Theorem 3 can be done above any low r.e. degree, i.e.

$$
\begin{aligned}
\forall \mathbf{d} \text { low } \forall \mathbf{a}> & \mathbf{d} \exists \mathbf{a}_{0}, \mathbf{a}_{1} \\
& \left(\mathbf{d}<\mathbf{a}_{0}, \mathbf{a}_{1}<\mathbf{a}, \mathbf{a}=\mathbf{a}_{0} \cup \mathbf{a}_{1} \text { and } \mathbf{a}_{0} \cap \mathbf{a}_{1} \text { does not exist }\right) .
\end{aligned}
$$

In (27) the assumption that $\mathbf{d}$ is low cannot be omitted: Lachlan [6] has shown that, in general, splitting and density cannot be combined. We can prove, however, that the pairs of r.e. degrees without infimum are dense in the r.e. degrees.

THEOREM 4. Let r.e. degrees $\mathbf{d}<\mathbf{c}$ be given. Then there are $\mathbf{a}_{0}$ and $\mathbf{a}_{1}$ such that $\mathbf{d}<\mathbf{a}_{0}, \mathbf{a}_{1}<\mathbf{c}$, and $\mathbf{a}_{0} \cap \mathbf{a}_{1}$ does not exist.

The proof of Theorem 4, which combines the construction of a pair of degrees without infimum with infinite injury priority arguments, requires some definitions from Soare [9]:

Given an r.e. set $X$ and an effective enumeration $\left\langle X_{s}: s<\omega\right\rangle$ of $X$ such that $\forall s\left(X_{s+1}-X_{s} \neq \varnothing\right), x_{s+1}$ denotes the least element of $X_{s+1}-X_{s}$ and $x_{0}=0$. A stage $t$ is a true stage of $X$ (with respect to $\left\langle X_{s}: s<\omega\right\rangle$ ) if $X_{t} \uparrow x_{t}=X \uparrow x_{t}$. A variant of the standard recursive approximation $\left\langle\{e\}_{s}^{X^{\prime}}: s<\omega\right\rangle$ to $\{e\}^{X}$ and the use function is defined by

$$
\{\hat{e}\}_{s}^{X_{s}}(x)= \begin{cases}\{e\}_{s}^{X_{s}}(x) & \text { if }\{e\}_{s}^{X_{s}}(x) \text { is defined and } u\left(e, X_{s}, x, s\right) \leqslant x_{s}, \\ \text { undefined } & \text { otherwise, }\end{cases}
$$


and

$$
\hat{u}\left(e, X_{s}, x, s\right)= \begin{cases}u\left(e, X_{s}, x, s\right) & \text { if }\{\hat{e}\}_{s}^{X_{s}}(x) \downarrow, \\ 0 & \text { otherwise, }\end{cases}
$$

respectively. The crucial property of this modified approximation is that for true $t$, a convergent computation $\{\hat{e}\}_{t}^{X_{t}}(x)$ is correct, i.e. $\{\hat{e}\}_{t}^{X_{t}}(x)=\{e\}^{X}(x), \hat{u}\left(e, X_{t}, x, t\right)=$ $u(e, X, x)$ and $X_{t} \uparrow \hat{u}\left(e, X_{t}, x, t\right)=X \uparrow \hat{u}\left(e, X_{t}, x, t\right)$ (see Soare [9, p. 518]).

Proof of Theorem 4. Let r.e. degrees $\mathbf{d}<\mathbf{c}$ be given. By density w.l.o.g. we may assume $\mathbf{d} \neq \mathbf{0}$. Choose r.e. sets $C \in \mathbf{c}, D \in \mathbf{d}$ and effective enumerations $\left\langle C_{s}: s\langle\omega\rangle\right.$ and $\left\langle D_{s}: s\langle\omega\rangle\right.$ of $C$ and $D$, respectively, such that $D \subseteq \omega^{(0)}, C^{(0)}=D, D_{0}=\varnothing$ and $\forall s\left(D_{s+1}-D_{s} \neq \varnothing\right)$. We construct r.e. sets $A_{0}$ and $A_{1}$ such that $\mathbf{a}_{0}=\operatorname{deg} A_{0}$ and $\mathbf{a}_{1}=\operatorname{deg} A_{1}$ have the desired properties.

To ensure $\mathbf{d} \leqslant \mathbf{a}_{0}, \mathbf{a}_{1}$ we set $A_{0}^{(0)}=A_{1}^{(0)}=D$. In general, we will have that $A_{j}^{(f)}$, $f<\omega, j \leqslant 1$, is recursive or recursive in $D$, and $A_{0}^{(f)}=A_{1}^{(f)}$, or one of them is empty, and we can decide which of them occurs. Hence for $A=A_{0} \cup A_{1}$ and $\mathbf{a}=\operatorname{deg} A$, $\mathbf{a}=\mathbf{a}_{0} \cup \mathbf{a}_{1}$. To guarantee $\mathbf{a} \leqslant \mathbf{c}$, we will make $A^{(f)} \leqslant_{T} C C$-uniformly in $f$ by strategies which depend on the type of requirement connected with $A^{(f)}$. For rows connected with the requirements which ensure that $\mathbf{a}_{0} \cap \mathbf{a}_{1}$ does not exist, we use the (delayed) permitting method. The hereby imposed restraints on $A$ interfere with our strategy to meet these requirements, and we succeed only if $C \$_{T} A$. Hence we have to meet the requirements

$$
\mathrm{R}_{3 e}: C \neq\{e\}^{A}
$$

for all $e \geqslant 1$ (since for some $e \geqslant 1,\{e\}^{A}=\{0\}^{A}$, we may omit $e=0$ ). To make $\mathbf{a}_{0}$ and $\mathbf{a}_{1}$ incomparable, we meet the requirements

$$
\mathrm{R}_{3(2 e+j)+1}: A_{j} \neq\{e\}^{A_{1-j}}
$$

for all $e<\omega$ and $j \leqslant 1$. Then to ensure that $\mathbf{a}_{0} \cap \mathbf{a}_{1}$ does not exist, it suffices to make, for some $j \leqslant 1, \mathbf{a}_{j}$ a minimal upper bound for $\left\{\mathbf{x}: \mathbf{x} \leqslant \mathbf{a}_{0}, \mathbf{a}_{1}\right\}$. For the sake of symmetry, we do this for both $\mathbf{a}_{0}$ and $\mathbf{a}_{1}$ : we construct (not necessarily r.e.) sets $E_{e}^{j}$, $j \leqslant 1, e=\left\langle e_{0}, e_{1}\right\rangle$, for which we meet the requirements

$$
\mathrm{R}_{3\langle 2 e+j, i\rangle+2}:\left(W_{e_{0}}=\left\{e_{1}\right\}^{A_{j}} \text { and } A_{j} k_{T} W_{e_{0}}\right) \rightarrow E_{e}^{j} \neq\{i\}^{W_{e_{0}}},
$$

and for which we ensure

$$
\begin{aligned}
& E_{e}^{j} \leqslant_{T} A_{1-j}, \\
& W_{e_{0}}=\left\{e_{1}\right\}^{A_{j}} \rightarrow E_{e}^{j} \leqslant_{T} A_{j} .
\end{aligned}
$$

That this implies $\mathbf{a}_{j}, j \leqslant 1$, is a minimal upper bound for $\left\{\mathbf{x}: \mathbf{x} \leqslant \mathbf{a}_{0}, \mathbf{a}_{1}\right\}$ is shown as follows: For a contradiction assume there is an r.e. degree $\mathbf{v}<\mathbf{a}_{j}$ such that $\forall \mathbf{x} \leqslant \mathbf{a}_{0}$, $\mathbf{a}_{1}(\mathbf{x} \leqslant \mathbf{v})$. Then we can choose $e=\left\langle e_{0}, e_{1}\right\rangle$ such that $W_{e_{0}} \in \mathbf{v}$ and the premises of $\mathrm{R}_{3\langle 2 e+j, i\rangle+2}$ and (29) hold. It follows that $\operatorname{deg} E_{e}^{j} \leqslant \mathbf{v}$ and $\operatorname{deg} E_{e}^{j} \leqslant \mathbf{a}_{0}, \mathbf{a}_{1}$. Though $\operatorname{deg} E_{e}^{j}$ is not necessarily r.e. by Lachlan [5, Lemma 18], there is an r.e. degree e such that $\operatorname{deg} E_{e}^{j} \leqslant \mathbf{e} \leqslant \mathbf{a}_{0}, \mathbf{a}_{1}$, which gives the desired contradiction. In the construction of $E_{e}^{j}$ a number put into $E_{e}^{j}$ at some stage can be extracted from $E_{e}^{j}$ at a later stage. 
For a fixed number, however, this can happen only finitely often. Hence with $E_{e, s}^{j}$ denoting the numbers put into $E_{e}^{j}$ at some stage $\leqslant s$ and not extracted from $E_{e}^{j}$ at a later stage $\leqslant s, E_{e}^{j}=\lim _{s} E_{e, s}^{j}$.

We say a number $f \geqslant 1$ is of type $i, i \leqslant 2$, if $f=3 e+i$ for some $e$. A requirement is of type $i$ if its index is. In the following $l, k, m$ stand for numbers of type $0,1,2$, respectively.

For meeting requirements of type 0 we use the preservation strategy, for that of type 1 the preservation and coding strategies of Sacks (see Soare [9, p. 525]). For this sake we need the following functions.

$$
\begin{aligned}
& l(3 e, s)=\max \left\{x: \forall y<x\left(C_{s}(y)=\{\hat{e}\}_{s}^{A_{s}}(y)\right)\right\}, \\
& l(3(2 e+j)+1, s)=\max \left\{x: \forall y<x\left(A_{j, s}(y)=\{\hat{e}\}_{s}^{A_{1-j . s}}(y)\right)\right\} \\
& m(3 e, s)=\max \{x: \exists t \leqslant s(x \leqslant l(3 e, t) \text { and } \\
& \left.\forall y<x\left(A_{t} \uparrow \hat{u}\left(e, A_{t}, y, t\right)=A_{s} \uparrow \hat{u}\left(e, A_{t}, y, t\right)\right)\right\}, \\
& m(3(2 e+j)+1, s) \\
& =\max \{x: \exists t \leqslant s(x \leqslant l(3(2 e+j)+1, t) \text { and } \\
& \left.\left.\forall y<x\left(A_{1-j, t} \backslash \hat{u}\left(e, A_{1-j, t}, y, t\right)=A_{1-j, s} \uparrow \hat{u}\left(e, A_{1-j, t}, y, t\right)\right)\right)\right\} \\
& \text { (modified length function), } \\
& r(3 e, s)=\max \left\{\hat{u}\left(e, A_{s}, x, s\right): x \leqslant m(3 e, s)\right\}, \\
& r(3(2 e+j)+1, s)=\max \left\{\hat{u}\left(e, A_{1-j, s}, x, s\right): x \leqslant m(3(2 e+j)+1, s)\right\}
\end{aligned}
$$

(restraint function).

(We will define $r(3 e+2, s)$ as a part of the construction below. $l(3 e+2, s)$ and $m(3 e+2, s)$ are undefined.)

Conditions (28) and (29) are satisfied using markers and the extended marker concept of the proof of Theorem 1, respectively. Let $\gamma_{j}$ be the standard marker of $A_{j}$, and for $e=\left\langle e_{0}, e_{1}\right\rangle$ and $x=\left\langle x_{0}, x_{1}\right\rangle$ define

$$
f_{e}^{j}(x, s)=\left\langle x_{0}, x_{1}, \max \left\{\bar{u}\left(e_{1}, A_{j, t}, y, t\right): y \leqslant \gamma_{j}(x, s) \text { and } t \leqslant s\right\}\right\rangle,
$$

where

$$
\begin{aligned}
\bar{u}\left(e_{1}, A_{j, s}, y, s\right) & =\hat{u}\left(e_{1}, A_{j, s}, y, s\right) \quad \text { if }\left\{\hat{e}_{1}\right\}_{s}^{A_{j, s}}(y) \downarrow \\
& =s \quad \text { otherwise. }
\end{aligned}
$$

Note that the function $f_{e}^{\jmath}$ has the following properties: It is nondecreasing in both arguments; $\left(f_{e}^{j}(x, s)\right)_{0}=(x)_{0}$; for any $x \neq y, s$ and $t, f_{e}^{j}(x, s) \neq f_{e}^{j}(y, t)$; and if $W_{e_{0}}=\left\{e_{1}\right\}^{A_{j}}$ then $f_{e}^{j}(x)=\lim _{s} f_{e}^{j}(x, s)$ is a total $A_{j}$-recursive function. Hence, to satisfy (28) and (29) it suffices to ensure

$$
\forall x \forall s\left(A_{1-j, s} \mid \gamma_{1-j}(x)=A_{1-j} \uparrow \gamma_{1-j}(x) \rightarrow E_{e, s}^{j} \backslash x=E_{e}^{j} \uparrow x\right)
$$

and

$$
W_{e_{0}}=\left\{e_{1}\right\}^{A_{j}} \rightarrow \forall x, s\left(A_{j, s} \uparrow f_{e}^{j}(x)=A_{j} \uparrow f_{e}^{j}(x) \rightarrow E_{e, s}^{j} \backslash x=E_{e}^{j} \uparrow x\right) .
$$


The basic strategy for satisfying the requirements of type 2 is similar to that used for like requirements in the preceding proofs. Roughly speaking, to meet $\mathbf{R}_{m}$, where $m=3\langle 2 e+j, i\rangle+2, e=\left\langle e_{0}, e_{1}\right\rangle, j \leqslant 1$, we wait for a number $x$ and a stage $s$ such that $E_{e, s}^{j}(x)=\{i\}_{s}^{W_{e} e_{s, s}(x), W_{e_{0}, s}}$ agrees with $\left\{\hat{e}_{1}\right\}_{s}^{A_{j, s}}$ on all numbers $y$ used in the computation $\{i\}_{s}^{W_{e}, s}(x)$, and $f_{e}^{j}(x, s)$ is not used in a computation $\left\{\hat{e}_{1}\right\}_{s}^{A_{j}, s}(y)$ for such a $y$. Then we set $E_{e, s+1}^{j}(x)=1-E_{e, s}^{j}(x)$ and try to preserve $\{i\}_{s}^{W_{e, s}(x)}$ by imposing an appropriated restraint on $A_{j}$. In order to satisfy (30) and (31) we put a number $z \leqslant \gamma_{1-j}(x, s)$ into $A_{1-j}$ and $f_{e}^{j}(x, s)$ into $A_{j}$. (In the actual construction we require $\gamma_{1-j}(x, s) \geqslant f_{e}^{j}(x, s)$; we then put $f_{e}^{j}(x, s)$ in both $A_{0}$ and $A_{1}$, thus ensuring $A_{0}^{(m)}=A_{1}^{(m)}$.) There are the following three obstacles to this procedure.

The first obstacle is that $R_{m}$ has to obey the restraints imposed by higher priority requirements, and these restraints can be unbounded now. It is necessary for satisfying $\mathbf{R}_{m}$ that at certain stages these restraints drop back simultaneously, i.e.

$$
\lim \inf _{s} \max \{r(f, s): f<m\}<\omega .
$$

For type 0 and 1 requirements we have the following: Let $T^{f}\left(T_{j}^{f}\right)$ be the set of true stages of $A^{(<f)}\left(A_{j}^{(<f)}\right)$ with respect to $\left\{\left(A_{s}\right)^{(<f)}: s<\omega\right\}\left(\left\{\left(A_{j, s}\right)^{(<f)}: s<\omega\right\}\right)$. Then, for $l<m, \lim _{t \in T^{m}} r(l, t)<\omega$ and, for $k=3(2 e+j)+1<m, \lim _{t \in T_{i-j}^{m}} r(k, t)<\omega$ will exist. To obtain infinitely many simultaneous "windows", we require $T^{m} \cap T_{0}^{m}$ $\cap T_{1}^{m}$ to be infinite. For this sake we consider the sets

$$
T_{D}^{f}=\left\{s: s \in T^{f} \text { and } a_{s}^{f} \in D_{s}\right\} \quad(f \geqslant 1),
$$

where $a_{0}^{f}=0$ and $a_{s+1}^{f}=\mu x\left(x \in A_{s+1}^{(<f)}-A_{s}^{(<f)}\right)$. Since, by construction, $\forall s\left(D_{s}=\right.$ $\left.A_{s}^{(0)}=A_{0, s}^{(0)}=A_{1, s}^{(0)}\right), T_{b} f \subseteq T^{f} \cap T_{0}^{f} \cap T_{f}^{f}$, and our assumption that $D$ is nonrecursive will imply $T_{D}^{f}$ is infinite. Finally we will see that, for any $m^{\prime}<m$ of type 2, $\lim _{t \in T_{D}^{m}} r\left(m^{\prime}, t\right)<\omega$ will exist. So $\lim _{t \in T_{D}^{m}} \max \{r(f, t): f<m\}<\omega$ will exist.

The second obstacle is that higher priority requirements can act infinitely often and the given set $D$ is a part of $A$. So there are infinitely many numbers enumerated in $A$ which do not have to obey the restraints imposed by $\mathrm{R}_{m}$. Such numbers can destroy our attempt to preserve a computation $\{i\}_{s}^{W_{e 0 . s}(x)}$ by restraining certain numbers from $A$. We prevent this from constantly happening by allowing $\mathrm{R}_{m}$ to be attacked by the same number $x$ more than once.

The third obstacle is that we are required to make $A$ recursive in $C$. Hence if we attack $\mathrm{R}_{m}$ as described above and put $z$ and $f_{j}^{e}(x, s)$ into $A_{1-j}$ and $A_{j}$, respectively, then we want these numbers permitted by $C$. It can happen, however, that $C$ never permits at a stage at which the higher priority restraints drop back. To overcome this difficulty we allow a delay in the permitting: If $C$ permits a number $y$ at a stage $s$ then this permitting is valid until the next stage $t+1$ such that $t \in T_{D}^{m}$. On the one hand this guarantees

$$
\forall m \forall x \in \omega^{(m)} \forall t \in T_{D}^{m}\left(C_{t} \uparrow x=C \uparrow x \rightarrow A_{t+1}(x)=A(x)\right),
$$

and thus $A \leqslant_{T} C$, since we will show $T_{D}^{m} \leqslant_{T} C$ uniformly in $m$ (i.e. the delay is $C$-recursive). On the other hand this delay guarantees that all permittings by $C$ happen at $T_{D}^{m}$-stages, i.e. - as outlined above-at stages with minimal restraints. 
The above considerations lead to the following definition:

Requirement $\mathbf{R}_{m}$, where $m=3\langle 2 e+j, i\rangle+2, e=\left\langle e_{0}, e_{1}\right\rangle$ and $j \leqslant 1$, requires attention at stage $s+1$ if there is an $x \in \omega^{(m)}$ such that

$$
r(m, s)=0 \text {, }
$$

$$
\begin{aligned}
& E_{e}^{j}(x)=\{i\}_{s}^{W_{e_{0}, s} s}(x), \\
& \gamma_{j}(x, s) \geqslant u, \quad \text { where } u=u\left(i, W_{e_{0}, s}, x, s\right), \\
& W_{e_{0}, s}\left|u=\left\{\hat{e}_{1}\right\}_{s}^{A_{j, s}}\right| u, \\
& f_{e}^{j}(x, s) \geqslant \max \{r(f, s): f<m\}, \\
& \gamma_{1-j}(x, s) \geqslant f_{e}^{j}(x, s) \text { and } f_{e}^{j}(x, s) \notin A_{s}, \\
& \text { there is a stage } t \leqslant s \text { such that }
\end{aligned}
$$

(32.7.1) $\exists y<f_{e}^{j}(x, s)\left(y \in C_{t+1}-C_{t}\right)$ and

(32.7.2) $\forall v\left(t \leqslant v<s \rightarrow a_{v}^{m} \notin D_{v}\right.$ or $\left.A_{v}^{(<m)}\left|a_{v}^{m} \neq A_{s}^{(<m)}\right| a_{v}^{m}\right)$.

We can now state the construction.

Stage 0. For all $m, r(m, 0)=0$.

Stage $s+1$. The stage consists of four steps. Numbers are put into or extracted from the sets under construction only during the first three steps. At the end of Step 1 we define a temporary restraint function $\hat{r}(f, s)$ valid for Step 3. The final restraint function $r(f, s+1)$ for stage $s+1$ is defined in Step 4.

Step 1 (Attacking type 2 requirements). Choose the least $m$ such that $\mathbf{R}_{m}$ requires attention, say $m=3\langle 2 e+j, i\rangle+2$ and $x$ is the least element of $\omega^{(m)}$ which satisfies (32.1)-(32.7). If $x$ is in $E_{e, s}^{j}$ extract $x$ from $E_{e}^{j}$; otherwise put $x$ into $E_{e}^{j}$. In either case put $f_{e}^{j}(x, s)$ into $A_{0}$ and $A_{1}$ and define

$$
\hat{r}(f, s)= \begin{cases}\max \left\{\hat{u}\left(e_{1}, A_{j, s}, y, s\right): y<u\left(i, W_{e_{0} . s}, x, s\right)\right\} & \text { if } f=m, \\ r(f, s) & \text { otherwise. }\end{cases}
$$

If no requirement $\mathbf{R}_{m}$ requires attention, set $\hat{r}(f, s)=r(f, s)$ for all $f$.

Step 2 (Coding $D$ into $A$ ). For all $x \in D_{s+1}-D_{s}$, put $x$ into $A_{0}$ and $A_{1}$.

Step 3 (Coding strategy for type 1 requirements). For $l=3(2 e+j)+1, j \leqslant 1$, $x<\omega$ and $t \leqslant s$ : If

$$
x \in C_{s},\langle l, x, t\rangle \geqslant \max \{\hat{r}(f, s): f<l\}
$$

and

$$
\forall v(t \leqslant v \leqslant s \rightarrow x \leqslant m(l, v))
$$

put $\langle l, x, t\rangle$ into $A_{j}$.

Step 4 (Preservation). For all $m=3\langle 2 e+j, i\rangle+2, j \leqslant 1$, define

$$
r(m, s+1)= \begin{cases}\hat{r}(m, s) & \text { if } A_{j, s+1} \uparrow \hat{r}(m, s)=A_{j, s}\lceil\hat{r}(m, s), \\ 0 & \text { otherwise. }\end{cases}
$$

Define $r(k, s+1), r(l, s+1)$ and the other auxiliary functions as described above. 
This completes the construction. To show that it is correct, we prove a series of lemmas. We start with some simple facts:

The construction is effective; so $A_{0}$ and $A_{1}$ are r.e. For any $e, x<\omega$ and $j \leqslant 1$, $\lim _{s} E_{e, s}^{j}(x)$ exists, since by (32.6) $x$ can be inserted in or extracted from $E_{e}^{j}$ at at most $\gamma_{1-j}(x)$ stages. Hence $E_{e}^{j}$ is well defined. Moreover, whenever $E_{e, s}^{j}(x) \neq$ $E_{e, s+1}^{j}(x), f_{e}^{j}(x, s)$ is enumerated in $A_{0}$ and $A_{1}$ at stage $s+1$. So, by (32.6), (30) and (31) hold, i.e. conditions (28) and (29) are satisfied by the constructed sets.

For the sake of requirement $\mathrm{R}_{f}$ only elements of $\omega^{(f)}$ are put into $A_{0}$ and $A_{1}$. If $f$ is of type $0, \mathrm{R}_{f}$ does not contribute any elements to $A_{0}$ or $A_{1}$; i.e. for any $k$, $A_{0}^{(k)}=A_{1}^{(k)}=\varnothing$. If $f=3(2 e+j)+1, j \leqslant 1$, then $A_{1-j}^{(f)}=\varnothing$, and if $f$ is of type 2 , then $A_{0}^{(f)}=A_{1}^{(f)}$. Finally, by Step 2 of stage $s+1, A_{0}^{(0)}=A_{1}^{(0)}=D$. From all this we conclude that $\mathbf{d} \leqslant \mathbf{a}_{0}, \mathbf{a}_{1}$ and $\mathbf{a}_{0} \cup \mathbf{a}_{1}=\mathbf{a}$.

Note that, by Step 2 of stage $s+1, A_{j, s+1}^{(<f)} \neq A_{j, s}^{(<f)}$ for any $f \geqslant 1, s<\omega$ and $j \leqslant 1$. So the numbers $a_{j, s}^{f}\left(a_{s}^{f}\right)$, where $a_{j, 0}^{f}=0$ and $a_{j, s+1}^{f}=\mu x\left(x \in A_{j, s+1}^{(<f)}-A_{j, s}^{(<f)}\right)$ $\left(a_{0}^{f}=0\right.$ and $\left.a_{s+1}^{f}=\mu x\left(x \in A_{s+1}^{(<f)}-A_{s}^{(<f)}\right)\right)$ are well defined. Recall that $T_{j}^{f}\left(T^{f}\right)$ denotes the set of true stages of $A_{j}^{(<f)}\left(A^{(<f)}\right)$, and $T_{b}^{f}=\left\{s: s \in T^{f}\right.$ and $\left.a_{s}^{f} \in D_{s}\right\}$. Note that for $e \leqslant f, T_{D}^{f} \subseteq T_{D}^{e}$ and, since $D=A_{0}^{(0)}=A_{1}^{(0)}, T_{D}^{f} \subseteq T_{0}^{f}, T_{1}^{f}, T^{f}$.

Lemma 11. Let $m, e, i, f, t<\omega$ and $j \leqslant 1$ be given such that $m=3\langle 2 e+j, i\rangle+2$, $f \geqslant m, t \in T_{j}^{f}$ and $r(m, t)>0$ or $\hat{r}(m, t)>0$. Then

(i) $\forall s>t(\hat{r}(m, t)=r(m, s)=\hat{r}(m, s)>0)$,

(ii) $A^{(m)}$ is finite, and

(iii) $\mathrm{R}_{m}$ is met.

Proof. Since $t \in T_{j}^{f}, A_{j, t}^{(<f)} \uparrow a_{j, t}^{f}=A_{j}^{(<f)} \uparrow a_{j, t}^{f}$, and since $a_{j, t} \leqslant a_{j, t}^{f}$ and $m \leqslant f$, this implies

$$
A_{j, t}^{(<m)} \uparrow a_{j, t}=A_{j}^{(<m)} \uparrow a_{j, t} .
$$

If $r(m, t)>0$, then by Step 4 of stage $t, A_{j, t} \uparrow r(m, t)=A_{j, t-1} \backslash r(m, t)$, i.e. $r(m, t)$ $\leqslant a_{j, t}$, and $\hat{r}(m, t)=r(m, t)$, since by (32.1) $\mathrm{R}_{m}$ does not require attention at stage $t+1$. If $r(m, t)=0$, then $\mathrm{R}_{m}$ is active at Step 1 of stage $t+1$. Hence for some $x$,

$$
\hat{r}(m, t)=\max \left\{\hat{u}\left(e_{1}, A_{j, s}, y, s\right): y<u\left(i, W_{e_{0}, s}, x, s\right)\right\} \leqslant a_{j, t} .
$$

So in either case, $0<\hat{r}(m, t) \leqslant a_{j, t}$, and therefore by (33),

$$
A_{j, t}^{(<m)} \uparrow \hat{r}(m, t)=A_{j}^{(<m)} \uparrow \hat{r}(m, t) .
$$

Since for $\hat{r}(m, s)>0, \hat{r}(m, s+1) \neq \hat{r}(m, s)$ only if a number less than $\hat{r}(m, s)$ enters $A_{j}$ at stage $s+1$, and since no such number can enter $A_{j}^{(\geqslant m)}$ at stage $s+1$, it follows by induction on $s \geqslant t$ that

$$
\begin{aligned}
& \forall s>t(\hat{r}(m, s)=r(m, s)=\hat{r}(m, t)>0 \\
& \left.\quad \text { and } A_{j, s} \mid \hat{r}(m, t)=A_{j, s-1} \backslash \hat{r}(m, t)\right) .
\end{aligned}
$$

This implies (i). (ii) immediately follows from (i), since a number can enter $A^{(m)}$ only at stages $s+1$ where $\mathbf{R}_{m}$ is active, i.e. where $0=r(m, s) \neq \hat{r}(m, s)$. To prove (iii), 
we fix the least $s^{\prime} \leqslant t$ s.t.

$$
\forall s\left(s^{\prime} \leqslant s \leqslant t \rightarrow \hat{r}(m, s)>0\right) .
$$

Then, by induction on $s$ with $s^{\prime} \leqslant s \leqslant t$ and by (34),

$$
\forall s>s^{\prime}\left(\hat{r}(m, s)=r(m, s)=\hat{r}\left(m, s^{\prime}\right)>0\right)
$$

and

$$
A_{j, s^{\prime}} \uparrow \hat{r}\left(m, s^{\prime}\right)=A_{j} \uparrow \hat{r}\left(m, s^{\prime}\right) .
$$

Moreover, $\mathrm{R}_{m}$ is active at stage $s^{\prime}+1$; i.e., there is an $x \in \omega^{(m)}$ such that

$$
\begin{aligned}
& E_{e, s^{\prime}+1}^{j}(x) \neq\{i\}^{W_{e_{0}, x^{\prime}}}(x), \\
& W_{e_{0}, s^{\prime}}\left|u\left(i, W_{e_{0}, s^{\prime}}, x, s^{\prime}\right)=\left\{\hat{e}_{1}\right\}_{s^{\prime}, u^{\prime}}^{A_{1}}\right| u\left(i, W_{e_{0}, s^{\prime}}, x, s^{\prime}\right), \quad \text { and } \\
& \hat{r}\left(m, s^{\prime}\right)=\max \left\{\hat{u}\left(e_{1}, A_{j, s^{\prime}}, y, s^{\prime}\right): y<u\left(i, W_{e_{0}, s^{\prime}}, x, s^{\prime}\right)\right\} .
\end{aligned}
$$

By (35), $\mathbf{R}_{m}$ does not require attention after stage $s^{\prime}+1$ and therefore $E_{c}^{j}(x)=$ $E_{e, s^{\prime}+1}^{j}(x)$. By (36),

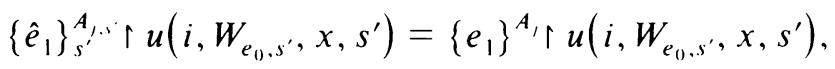

thus $W_{e_{0}} \neq\left\{e_{1}\right\}^{A}$, or $\{i\}^{W_{c_{0}, \cdot} \cdot(x)}=\{i\}^{W_{c_{0}}}(x)$. In either case $\mathrm{R}_{m}$ is met.

LEMMA 12. For all $m$,

$$
F_{m}=\left\{y \in A^{(m)}: \exists s\left(y \in A_{s+1}^{(m)}-A_{s}^{(m)} \text { and } A_{s}^{(<m)} \uparrow y=A^{(<m)} \uparrow y\right)\right\}
$$

is finite.

Proof. Let $m=3\langle 2 e+j, i\rangle+2, j \leqslant 1$, be given and assume $y \in F_{m}$. Then there is a stage $s$ such that $y \in A_{s+1}^{(m)}-A_{s}^{(m)}$ and $A_{s}^{(<m)} \mid y=A^{(<m)} \uparrow y$. Hence $\mathbf{R}_{m}$ receives attention at stage $s+1$ via some $x$ and $0<\hat{r}(m, s) \leqslant f_{e}^{j}(x, s)=y$. As in the proof of Lemma 11 we can conclude that for all $s^{\prime}>s, \hat{r}\left(m, s^{\prime}\right)=r\left(m, s^{\prime}\right)=\hat{r}(m, s)>0$; i.e., $\mathrm{R}_{m}$ does not require attention after stage $s+1$, therefore $F_{m} \subseteq A^{(m)}=A_{s+1}^{(m)}$ is finite.

LeMma 13. For all $f<\omega$ the following hold:

(a) If $f$ is of type 0 or $1, \mathrm{R}_{f}$ is met.

(b) If $f$ is of type 0 or $1, A^{(f)}$ is recursive. If $f=0$ or $f$ of type $2, A^{(f)} \leqslant_{T} D$.

(c) The set $T_{D}^{+1}$ is infinite.

(d) For all $1 \leqslant f^{\prime} \leqslant f+1$ :

$$
\begin{aligned}
& \lim _{t \in T_{D}^{f+1}} r\left(f^{\prime}, t\right)=\lim _{t \in T_{D}^{\prime+1}} \hat{r}\left(f^{\prime}, t\right)<\omega \text { exists; } \\
& \forall t \in T_{D}^{f+1} \forall s \geqslant t\left(r\left(f^{\prime}, s\right) \geqslant r\left(f^{\prime}, t\right) \text { and } \hat{r}\left(f^{\prime}, s\right) \geqslant \hat{r}\left(f^{\prime}, t\right)\right) .
\end{aligned}
$$

Proof. By induction on $f$. For the inductive step fix $f$.

(a) We distinguish two cases.

Case 1: $f=3 e>0$. For a contradiction assume $\mathbf{R}_{f}$ is not met, i.e. $C=\{e\}^{A}$. Then, by Soare [9, Lemma 2.1], $C \leqslant_{T} I_{e}$, where

$$
I_{c}=\left\{x: \exists s\left(x \in A_{s+1}-A_{s} \text { and } x<r(f, s)\right\} .\right.
$$


By construction, $I_{e} \subseteq A^{(<f)}$, therefore $I_{e} \leqslant_{T} A^{(<f)}$. Since by inductive hypothesis (b), $A^{(<f)} \leqslant_{T} D$, this contradicts $D<{ }_{T} C$.

Case 2: $f=3(2 e+j)+1, j \leqslant 1$. For a contradiction assume $\mathbf{R}_{f}$ is not met, i.e. $A_{j}=\{e\}^{A_{1}}$. Then $\lim _{s} m(f, s)=\omega$. Hence

$$
M(x)=\mu s\left(\forall s^{\prime} \geqslant s\left(m\left(f, s^{\prime}\right) \geqslant x\right)\right)
$$

is a total function. By construction, for any $s$ such that $m(f, s) \geqslant x, s<M(x)$ iff $A_{1-j, s}^{(<f)}\left|u \neq A_{1-j}^{(<f)}\right| u$, where $u=\max \left\{\hat{u}\left(e, A_{1-j . s}, y, s\right): y<x\right\}$. Hence $M \leqslant_{T} A_{1-j}^{(<f)}$, thus, by inductive hypothesis, $M \leqslant_{T} D$. Note that for $s \geqslant M(x), r(f, s) \geqslant m(f, s)$ $\geqslant x$. Hence, by construction,

$$
\forall x\left(A_{1-j, M(x)}^{(\geqslant f)} \uparrow x=A_{1-j}^{(\geqslant f)} \uparrow x\right)
$$

(note that $A_{1-j}^{(f)}=\varnothing$ ). This implies $A_{1-j}^{(\geqslant f)} \leqslant_{T} D$, thus, by inductive hypothesis, $A_{1-j}$ is recursive in $D$. Since, by assumption, $A_{j} \leqslant_{T} A_{1-j}$, this implies $A={ }_{T} A_{0} \oplus A_{1} \leqslant_{T} D$.

Now let $r=\liminf _{s} \max \left\{r\left(f^{\prime}, s\right): f^{\prime}<f\right\}$. By inductive hypotheses (e) and (d), $r<\omega$. By construction, for $x \geqslant r, x \in C$ iff $\langle f, x, M(x)\rangle \in A_{j}$. Hence $C \leqslant_{T} M \oplus A_{j}$ $\leqslant_{T} A_{1-j} \oplus A_{j} \leqslant_{T} D$, a contradiction.

(b) We distinguish four cases.

Case 1: $f$ is of type 0 . Then $A^{(f)}=\varnothing$.

Case 2: $f$ is of type 1 . Say $f=3(2 e+j)+1, j \leqslant 1$. Then $A_{1-j}^{(f)}=\varnothing$, i.e. $A^{(f)}=A_{j}^{(f)}$. By (a) there is $p<\omega$ with $p=\liminf _{s} m(f, s)$. By inductive hypothesis, choose $r<\omega$ such that $r=\liminf _{s} \max \left\{r\left(f^{\prime}, s\right): f^{\prime}<f\right\}$, and fix a stage $s_{0}$ such that $A_{j, s_{0}} \uparrow p+1=A_{j} \uparrow p+1$ and $\forall s \geqslant s_{0}\left(\max \left\{r\left(f^{\prime}, s\right): f^{\prime}<f\right\} \geqslant r\right)$. Now for given $x$ and $s$, we can decide whether $\langle f, x, s\rangle \in A$ as follows: If $x>p$, find the least stage $s^{\prime} \geqslant s$ such that $m\left(f, s^{\prime}\right)=p$. Then $\langle f, x, s\rangle \in A$ iff $\langle f, x, s\rangle \in A_{s^{\prime}}$. If $x \leqslant p$, find the least stage $t \geqslant s, s_{0}$ s.t. $\max \left\{r\left(f^{\prime}, t\right): f^{\prime}<f\right\}=r$. Then $\langle f, x, s\rangle \in A$ iff $\langle f, x, s\rangle \in A_{t}$.

Case 3: $f$ is of type 2. By Lemma 12, fix $z$ such that $\forall y \in F_{t}(y<z)$, and a stage $s_{0}$ s.t. $A_{s_{0}}^{(f)} \uparrow z=A^{(f)} \uparrow z$. Then

$$
\forall x \forall s \geqslant s_{0}\left(A_{s}^{(<f)} \uparrow x=A^{(<f)}\left|x \rightarrow A_{s}^{(f)}\right| x=A^{(f)} \mid x\right) .
$$

Hence $A^{(f)} \leqslant_{T} A^{(<f)}$, therefore, by inductive hypothesis, $A^{(f)} \leqslant_{T} D$.

Case 4: $f=0$. Then $A^{(f)}=A^{(0)}=D$.

(c) For a contradiction assume $T_{b}^{+1}$ is finite, i.e., there are only finitely many $t \in T^{f+1}$ with $a_{t}^{f+1} \in A^{(0)}=D$. Since for $f^{\prime}$ of type $0, A^{\left(f^{\prime}\right)}=\varnothing$ and for $f^{\prime} \leqslant f$ of type 2 , by Lemma 12 , there are only finitely many $t \in T^{f+1}$ with $a_{t}^{f+1} \in A^{\left(f^{\prime}\right)}$, it follows that there is an $s_{0}$ such that

$$
\forall t>s_{0}\left(t \in T^{f+1} \rightarrow \exists l \leqslant f\left(l \text { of type } 1 \text { and } a_{t}^{f+1} \in A^{(l)}\right)\right) .
$$

Hence, for all $x>a_{s_{0}}^{f+1}$ and $s \geqslant s_{0}$,

$$
\cup\left\{A_{s}^{(l)}: l \leqslant f\right\}\left|x=\bigcup\left\{A^{(l)}: l \leqslant f\right\}\right| x \rightarrow A_{s}^{(<f+1)} \mid x=A^{(<f+1)} \uparrow x ;
$$

thus $A^{(<f+1)} \leqslant_{T} \cup\left\{A^{(l)}: l \leqslant f\right\}$. Since $D=A^{(0)} \leqslant A_{T} A^{(<f+1)}$ and, by inductive hypothesis and (b), $\cup\left\{A^{(l)}: l \leqslant f\right\}$ is recursive, this implies $D$ is recursive, contrary to our assumption. 
(d) For $1 \leqslant f^{\prime}<f+1$, (d) holds by inductive hypothesis, since for $f>0, T_{D}^{f} \supseteq$ $T_{D}^{f+1}$. Hence it suffices to consider $f^{\prime}=f+1$.

Case 1: $f+1$ is of type 0 . Then for $t \in T^{f+1}$, by induction on $s \geqslant t$,

$$
\begin{aligned}
\forall s \geqslant t(m(f+1, t) & \leqslant m(f+1, s), r(f+1, t) \leqslant r(f+1, s) \\
& \left.=\hat{r}(f+1, s) \text { and } A_{t} \uparrow r(f+1, t)=A_{s} \uparrow r(f+1, t)\right) .
\end{aligned}
$$

Since $T_{D}^{f+1} \subseteq T^{f+1}$, this implies (38). By (a) let $p=\mu x\left(C(x) \neq\{e\}^{A}(x)\right)$ and choose $t \in T^{f+1}$ such that $C_{t} \uparrow p+1=C \uparrow p+1$ and $m(f+1, t) \geqslant p$. Then

$$
\forall s \geqslant t\left(s \in T^{f+1} \rightarrow m(f+1, s) \leqslant m(f+1, t)\right) ;
$$

thus by definition of $r$ and (39),

$$
\forall s \geqslant t\left(s \in T^{f+1} \rightarrow \hat{r}(f+1, s)=r(f+1, s)=r(f+1, t)\right),
$$

which implies (37).

Case 2: $f+1$ is of type 1. Say $f+1=3(2 e+j)+1, j \leqslant 1$. Similar to Case 1 (consider stages in $T_{1-j}^{f+1}$ and note that $T_{D}^{f+1} \subseteq T_{1-j}^{f+1}$ ).

Case 3: $f+1$ is of type 2. Then the claim follows from Lemma 11 and $T_{D}^{f+1} \subseteq T_{j}^{f+1}$, $j \leqslant 1$.

Note that by Lemma 13(a), $C \$_{T} A$ and $\left.A_{0}\right|_{T} A_{1}$.

LEMMA 14. Let $m=3\langle 2 e+j, i\rangle+2, j \leqslant 1$ and $e=\left\langle e_{0}, e_{1}\right\rangle$ be given and assume $W_{e_{0}}=\left\{e_{1}\right\}^{A}$. Then

$$
\exists x \in \omega^{(m)}\left(f_{e}^{j}(x) \in A\right) \rightarrow \mathrm{R}_{m} \text { is met } .
$$

Proof. Assume $f_{e}^{j}(x) \in A, x \in \omega^{(m)}$. Then there is a last stage $s$ such that $\mathrm{R}_{m}$ receives attention via $x$ at stage $s+1$; i.e.,

$$
\begin{aligned}
& E_{e, s+1}^{j}(x) \neq\{i\}^{W_{e^{\prime}(p) \cdot}}(x) \downarrow, \\
& W_{e_{0}, s} \uparrow u\left(i, W_{e_{0}, s}, x, s\right)=\left\{\hat{e}_{1}\right\}_{s}^{A, s \uparrow} \mid u\left(i, W_{e_{0}, s}, x, s\right) \text {, } \\
& f_{e}^{j}(x)=f_{e}^{j}(x, s) \geqslant v \text {, }
\end{aligned}
$$

where $v=\max \left\{\hat{u}\left(e_{1}, A_{j, s}, y, s\right): y<u\left(i, W_{e_{0} . s}, x, s\right)\right\}$, and $f_{e}^{j}(x, s)$ is put into $A$ at stage $s+1$. To show that $\mathrm{R}_{m}$ is met, it suffices to show that $A_{j, s} \uparrow v=A_{j} \uparrow v$. If this is not the case, fix the least $t>s$ s.t. $A_{j, s} \uparrow v \neq A_{j, t} \uparrow v$. Then, by definition of $f_{e}^{j}$,

$$
\left.f_{e}^{j}(x, t)=\left\langle(x)_{0},(x)_{1}, t\right\rangle\right\rangle\left\langle(x)_{0},(x)_{1}, s\right\rangle \geqslant f_{e}^{j}(x, s),
$$

i.e. $f_{e}^{j}(x, s) \neq f_{e}^{j}(x)$, contrary to assumption.

Lemma 15. For all $m, \mathrm{R}_{m}$ is met.

Proof. Fix $m=3\langle 2 e+j, i\rangle+2, j \leqslant 1, e=\left\langle e_{0}, e_{1}\right\rangle$ and assume, for a contradiction, $\mathrm{R}_{m}$ is not met, i.e.

$$
\begin{aligned}
& W_{e_{0}}=\left\{e_{1}\right\}^{A,}, \\
& A_{j}{ }_{T} W_{e_{0}}, \\
& E_{e}^{j}=\{i\}^{W_{e^{\prime}}} .
\end{aligned}
$$


Since $T_{D}^{m} \subseteq T_{0}^{m} \cap T_{1}^{m}$, Lemma 11 implies every type 2 requirement $\mathrm{R}_{m^{\prime}}, m^{\prime} \leqslant m$, requires attention at at most finitely many stages $t+1$ such that $t \in T_{D}^{m}$. So we can choose $s_{0}$ such that

$$
\forall t \geqslant s_{0}\left(t \in T_{D}^{m} \rightarrow \mathrm{R}_{m} \text { does not require attention at stage } t+1\right) .
$$

In the following we will refute (43), thus obtaining the desired contradiction.

By Lemma 13, let

$$
r=\sup _{t \in T_{D}^{m}} \max \{r(f, t): f<m\}
$$

and define $Z=\left\{x \in \omega^{(m)}: x>r\right\}$. We will first show there is an $A_{j}$-recursive function $s(x)$ such that

$$
\forall x \in Z \forall s \geqslant s(x)\left(s \in T_{D}^{m} \rightarrow(32.1),\right. \text { (32.2), (32.4), (32.5) }
$$

and the second part of (32.6) hold).

Since $f_{e}^{j}(x, s) \geqslant x$ for any $x, s$,

$$
\forall x \in Z \forall s \in T_{D}^{m}\left(f_{e}^{j}(x, s)>\max \{r(f, s): f<m\}\right) .
$$

By (40) and (42), $E_{e}^{j} \leqslant{ }_{T} W_{e_{0}} \leqslant{ }_{T} A_{j}$ and

$$
\forall x\left(E_{e}^{j}(x)=\{i\}^{W_{e_{0}}}(x) \text { and } W_{e_{0}} \uparrow u\left(i, W_{e_{0}}, x\right)=\left\{e_{1}\right\}^{A_{j}} \uparrow u\left(i, W_{e_{0}}, x\right)\right) .
$$

So we can $A_{j}$-recursively compute a stage $s(x)$ such that

$$
E_{e, s(x)}^{j}(x)=\{i\}_{s(x)}^{W_{e_{0}, s(x)}}(x)
$$

and

$$
W_{e_{0}, s(x)} \mid u\left(i, W_{e_{0}, s(x)}, x, s(x)\right)=\left\{e_{1}\right\}_{s(x)}^{A_{j, s(x)} \mid} u\left(i, W_{e_{0}, s(x)}, x, s(x)\right)
$$

via correct computations; i.e.,

$$
\begin{aligned}
\forall x \forall s \geqslant s(x)\left(\{i\}_{s}^{W_{e_{0}, s}}(x)=\right. & \{i\}_{s(x)}^{W_{e_{0}, s(x)}}(x) \\
& \text { and } \left.u\left(i, W_{e_{0} . s}, x, s\right)=u\left(i, W_{e_{0}, s(x)}, x, s(x)\right)\right)
\end{aligned}
$$

and

$$
\begin{aligned}
\forall x \forall s \geqslant s(x)\left(W_{e_{0}, s} \backslash u\left(i, W_{e_{0}, s}, x, s\right)=\left\{e_{1}\right\}_{s}^{A_{j, s}} \backslash u\left(i, W_{e_{0}, s}, x, s\right)\right. \text { and } \\
\left.\quad \forall y<u\left(i, W_{e_{0}, s}, x, s\right)\left(u\left(e_{1}, A_{j, s}, y, s\right)=u\left(e_{1}, A_{j, s(x)}, y, s(x)\right)\right)\right) .
\end{aligned}
$$

Since, by (42), $f_{e}^{j}(x)=\lim _{s} f_{e}^{j}(x, s)$ is a total $A_{j}$-recursive function and since $f_{e}^{j}(x, s)$ is nondecreasing in $s$, we can $A_{j}$-recursively find a stage $s_{x}$ such that $f_{e}^{j}(x, s)=f_{e}^{j}(x)$ for $s \geqslant s_{x}$. W.l.o.g. we may assume $s_{x} \leqslant s(x)$, i.e.

$$
\forall x \forall s \geqslant s(x)\left(f_{e}^{j}(x, s)=f_{e}^{j}(x)\right) .
$$

This and Lemma 14 imply

$$
\forall x \in Z \forall s \geqslant s(x)\left(f_{e}^{j}(x, s) \notin A\right) .
$$

Since $E_{e, s+1}^{j}(x) \neq E_{e, s}^{j}(x)$ implies $f_{e}^{j}(x, s)$ is in $A_{s+1}$, we can conclude that

$$
\forall x \in Z \forall s \geqslant s(x)\left(E_{e . s}^{j}(x)=E_{e}^{j}(x)\right)
$$


and thus, by (42) and (46),

$$
\forall x \in Z \forall s \geqslant s(x)\left(E_{e, s}^{j}(x)=\{i\}_{s}^{\left.W_{c i, s}(x)\right)} .\right.
$$

Finally, since $\mathbf{R}_{m}$ is not met, Lemma 11 implies

$$
\forall t \in T_{D}^{m}(r(m, t)=0) .
$$

Facts (51), (50), (47), (45) and (49) imply (44) holds.

In the next step we define an infinite $A$-recursive subset $Z^{\prime \prime}$ of $Z$, and an $A$-recursive function $s^{\prime \prime}(x)$ such that

$$
\forall x \in Z^{\prime \prime} \forall s \geqslant s^{\prime \prime}(x)\left(s \in T_{D}^{m} \rightarrow(32.1) \text { - (32.6) hold }\right) .
$$

We first note that, by (42), $u\left(i, W_{e_{0}}, x\right)$ is a total $W_{e_{0}}$-recursive function. Hence, by (41) and Lemma 1, the set

$$
Z^{\prime}=\left\{x \in Z: \gamma_{j}(x)>u\left(i, W_{e_{01}}, x\right)\right\}
$$

is infinite. Moreover, $Z^{\prime} \leqslant_{T} A_{j} \oplus W_{e_{0}} \leqslant_{T} A_{j}$, and we can define an $A_{j}$-recursive function $s^{\prime}(x)$ such that $\forall x\left(s^{\prime}(x) \geqslant s(x)\right)$ and

$$
\forall x \in Z^{\prime} \forall s \geqslant s^{\prime}(x)\left(\gamma_{j}(x, s)>u\left(i, W_{e_{0}, s}, x, s\right)\right) .
$$

Now, since $Z^{\prime}$ is an infinite $A_{j}$-recursive set, since $f_{e}^{j}(x)$ is $A_{j}$-recursive, and since, by Lemma 13(a), $A_{1-j} \$_{T} A_{j}$, a second application of Lemma 1 shows that the set

$$
Z^{\prime \prime}=\left\{x \in Z^{\prime}: \gamma_{1-j}(x)>f_{e}^{j}(x)\right\}
$$

is infinite. $Z^{\prime \prime} \leqslant_{T} A_{0} \oplus A_{1}={ }_{T} A$ and there is an $A$-recursive function $s^{\prime \prime}$ such that

$$
\begin{aligned}
& \forall x\left(s^{\prime \prime}(x) \geqslant s^{\prime}(x) \geqslant s(x)\right) \text { and } \\
& \forall x \in Z^{\prime \prime} \forall s \geqslant s^{\prime \prime}(x)\left(\gamma_{1} j(x, s)>f_{e}^{j}(x, s)\right) .
\end{aligned}
$$

Now (52) follows from (54), (44), (53) and (55).

Since $Z^{\prime \prime}$ is infinite and $Z^{\prime \prime}, s^{\prime \prime} \leqslant_{T} A$,

$$
\forall x \in Z^{\prime \prime} \forall s>\max \left\{s^{\prime \prime}(x), s_{0}\right\}\left(C_{s} \uparrow x=C \uparrow x\right)
$$

implies $C \leqslant_{T} A$, contrary to Lemma 13(a). Hence we can fix $x, y, t$ such that $x \in Z^{\prime \prime}, y<x, y \in C_{t+1}-C_{t}$ and $t>\max \left\{s^{\prime \prime}(x), s_{0}\right\}$. Now if $s$ is the least stage $\geqslant t$ in $T_{D}^{m_{2}}$ then (32.7.1) and (32.7.2) hold (note that $x \leqslant f_{e}^{j}(x, s)$ ). So, by (52), $\mathbf{R}_{m}$ requires attention at stage $s+1$ contrary to (43).

Lemma $16 . A \leqslant_{T} C$.

Proof. Since $A^{(0)}=D \leqslant_{T} C$, it suffices to show that for $f>0, A^{(f)}$ is recursive in $C, C$-uniformly in $f$ (i.e. there is a $C$-recursive function $g$ s.t. $\left.A^{(f)}=\{g(f)\}^{C}\right)$. This is done by an effective induction. For the inductive step fix $f$. By inductive hypothesis we may use $C \oplus A^{(<f)}$ as oracle, and thus $C \oplus T_{D}^{f}$, since $T_{D}^{f} \leqslant_{T} A^{(<f)}$ uniformly in $f$.

Case 1: $f$ is of type 0 . Then $A^{(f)}=\varnothing$.

Case 2: $f$ is of type 1 . Say $f=3(2 e+j)+1, j \leqslant 1$. To $C \oplus T_{D}^{f}$-recursively decide whether a given $\langle f, x, t\rangle$ is in $A$, first check if $x \in C$. If not, $\langle f, x, t\rangle \notin A$. If so, choose $s$ with $x \in C_{s}$ and the least element $\left.s^{\prime}\right\rangle s, t$ of $T_{D}^{f}$. Then, by (38), $\langle f, x, t\rangle \in A$ iff $\langle f, x, t\rangle \in A_{s^{\prime}}$. 
Case 3: $f$ is of type 2. To decide whether a given $\langle f, x\rangle$ is in $A$, find the least stage $t \in T_{D}^{f}$ with $C_{t} \uparrow\langle f, x\rangle=C \uparrow\langle f, x\rangle$. Then by (32.7), $\langle f, x\rangle \in A$ iff $\langle f, x\rangle \in A_{t+1}$.

This completes the proof of Theorem 4.

Note that for $\operatorname{low}_{2} \mathbf{c}$, the above constructed degrees $\mathbf{a}_{0}$ and $\mathbf{a}_{1}$ form a minimal exact pair for some u.r.e. ascending sequence of r.e. degrees.

ACKNOWLEDGMENTS. Portions of this paper are contained in our dissertation [1], prepared while we were visiting the University of Connecticut and Cornell University in 1980-1981. We would iike to thank P. A. Fejer, M. Lerman, W. Maass, R. A. Shore and R. I. Soare for helpful suggestions and discussions during this period. The results of the paper were presented to the American Mathematical Society during a special session on Recursion Theory on November 1, 1980.

\section{REFERENCES}

1. K. Ambos-Spies, On the structure of the recursively enumerable degrees, Dissertation, Universität München, 1980.

2. An extension of the non-diamond theorem in classical and $\alpha$-recursion theory, J. Symbolic Logic 49 (1984).

3. S. B. Cooper, Minimal upper bounds for sequences of recursively enumerable degrees, J. London Math. Soc. 5 (1972), 445-450.

4. C. G. Jockusch, Three easy constructions of recursively enumerable sets, Logic Year 1979-80, Lectures Notes in Math., vol. 859, Springer-Verlag, Berlin and New York, 1981, pp. 83-91.

5. A. H. Lachlan, Lower hounds for pairs of r.e. degrees, Proc. London Math. Soc. (3) 16 (1966), $537-569$.

6. A r a recursively enumerable degree which will not split over all lesser ones, Ann. Math. Logic 9 (1975), 307-365.

7. Decomposition of recursively enumerable degrees, Proc. Amer. Math. Soc. 79 (1980), 629-634.

8. R. W. Robinson, Interpolation and embedding in the recursively enumerable degrees, Ann. of Math. (2) 93 (1971), 285-314.

9. R. I. Soare, The infinite injury priority method, J. Symbolic Logic 41 (1976), 513-530.

10. , Fundamental methods for constructing recursively enumerable degrees, Recursion Theory: Its Generalisations and Applications, London Math. Soc. Lecture Notes Ser. 45, Cambridge, 1980, pp. 1-51.

11. C. E. M. Yates, A minimal pair of r.e. degrees, J. Symbolic Logic 31 (1966), 159-168.

12. . On the degrees of index sets. II, Trans. Amer. Math. Soc. 135 (1969), 249-266.

Lehrstuhl Informatik II, Universität Dortmund, 4600 Dortmund 50, Postfach 50 05 00, West GERMANY 